\title{
An investigation of tuberculosis progression revealing the role of macrophages apoptosis via sensitivity and bifurcation analysis
}

\author{
Wenjing Zhang ${ }^{1}$ (D) $\cdot$ Leif Ellingson $^{1} \cdot$ Federico Frascoli ${ }^{2} \cdot$ Jane Heffernan $^{3}$ \\ Received: 18 December 2020 / Revised: 25 May 2021 / Accepted: 16 August 2021 / \\ Published online: 26 August 2021 \\ (c) The Author(s), under exclusive licence to Springer-Verlag GmbH Germany, part of Springer Nature 2021
}

\begin{abstract}
Mycobacterium tuberculosis infection features various disease outcomes: clearance, latency, active disease, and latent tuberculosis infection (LTBI) reactivation. Identifying the decisive factors for disease outcomes and progression is crucial to elucidate the macrophages-tuberculosis interaction and provide insights into therapeutic strategies. To achieve this goal, we first model the disease progression as a dynamical shift among different disease outcomes, which are characterized by various steady states of bacterial concentration. The causal mechanisms of steady-state transitions can be the occurrence of transcritical and saddle-node bifurcations, which are induced by slowly changing parameters. Transcritical bifurcation, occurring when the basic reproduction number equals to one, determines whether the infection clears or spreads. Saddle-node bifurcation is the key mechanism to create and destroy steady states. Based on these two steady-state transition mechanisms, we carry out two sample-based sensitivity analyses on transcritical bifurcation conditions and saddle-node bifurcation conditions. The sensitivity analysis results suggest that the macrophage apoptosis rate is the most sig-
\end{abstract}

$凶 \quad$ Wenjing Zhang

wenjing.zhang@ttu.edu

Leif Ellingson

leif.ellingson@ttu.edu

Federico Frascoli

ffrascoli@swin.edu.au

Jane Heffernan

jmheffer@yorku.ca

1 Department of Mathematics and Statistics, Texas Tech University, Broadway and Boston, Lubbock 79409-1042, TX, USA

2 Department of Mathematics, Faculty of Science, Engineering and Technology, Swinburne University of Technology, John St, 3122, Hawthorne, VIC, Australia

3 Department of Mathematics and Statistics, Centre for Disease Modelling, York University, 4700 Keele St, Toronto, ON M3J 1P3, Canada 
nificant factor affecting the transition in disease outcomes. This result agrees with the discovery that the programmed cell death (apoptosis) plays a unique role in the complex microorganism-host interplay. Sensitivity analysis narrows down the parameters of interest, but cannot answer how these parameters influence the model outcomes. To do this, we employ bifurcation analysis and numerical simulation to unfold various disease outcomes induced by the variation of macrophage apoptosis rate. Our findings support the hypothesis that the regulation mechanism of macrophage apoptosis affects the host immunity against tuberculosis infection and tuberculosis virulence. Moreover, our mathematical results suggest that new treatments and/or vaccines that regulate macrophage apoptosis in combination with weakening bacillary viability and/or promoting adaptive immunity could have therapeutic value.

Keywords In-host tuberculosis infection model · Disease progression · Sensitivity analysis and bifurcation analysis - Tuberculosis treatments.

Mathematics Subject Classification $34 \mathrm{C} 23 \cdot 34 \mathrm{C} 20 \cdot 34 \mathrm{C} 15 \cdot 37 \mathrm{~N} 25$

\section{Introduction}

Tuberculosis (TB), a disease caused by Mycobacterium tuberculosis (Mtb), remains the deadliest infectious disease in the world (Sud et al. 2006; Lin and Flynn 2010). According to a CDC (Centers for Disease Control and Prevention) report (WHO 2019), in the United States, the reactivation of latent Tuberculosis infection was a major cause for TB incidence in 2019. A detailed understanding of the most significant driving factors for TB disease progression is crucial for the improvement of the treatment strategies to help host fight against the invader pathogen.

To serve this motivation, we adopt an established in-host tuberculosis infection model that generates various disease outcomes. Since transcritical bifurcations determine the fate of infection and saddle-node bifurcations create multiple infected equilibrium solutions, we carry out a parameter sensitivity analysis on the conditions for transcritical and saddle-node bifurcations. We then further investigate model behavior through bifurcation analysis to examine how the macrophage apoptosis rate and other statistically significant parameters impact disease progression and examine how the mathematical results relate to the complexities of the disease dynamics. Finally, we utilize a 2-dimensional bifurcation analysis to explore how combination therapies can enhance macrophage apoptosis modulation therapy to inhibit bacillary viability and promote adaptive immunity.

Before we present our methodology and results, we provide a brief introduction of tuberculosis, including the various disease outcomes, in-host Mtb life cycles, and therapeutic strategies, in Sect. 1.1 and discuss the previous in-host Mtb mathematical modeling literature in Sect. 1.2. 


\subsection{TB epidemiology and immunology background}

Tuberculosis (TB), a disease caused by pathogenic bacterium species Mycobacterium tuberculosis (Mtb), has been spreading among humans for thousands of years (Donoghue et al. 2004). Despite the availability of effective TB treatments, TB continues claiming 1.5 million lives per year by infecting one quarter of the global population (Sud et al. 2006; Lin and Flynn 2010). A characteristic feature for Tuberculosis infection is that infected individuals experience various disease outcomes, including clearance, latent infection (LTBI), and active disease. Approximately 10\% of infected individuals clear the infection and another $10 \%$ progress to primary disease, while the majority of infected individuals become latently infected with no symptoms. Individuals with LTBI carry the risk of developing TB later in life. Slow TB progression through LTBI reactivation contributes approximately $80 \%$ of cases globally and threatens a TB re-emergence (Cooper 2009; Rich et al. 1951). A better understanding on TB progression, especially LTBI reactivation, can help to improve the efficacy of therapeutic strategies, and enhance both the innate and adaptive immune responses.

The life cycle of tuberculosis begins when aerosol droplets containing Mtb are inhaled by an uninfected individual. After inhalation, Mtb usually reach the alveoli of the lung, and are preferentially ingested by alveolar macrophages (Warner and Mizrahi 2007), then launch proliferation (Canetti 1955). This makes alveolar macrophages the main target cells for Mtb invasion (Gammack et al. 2005; Gideon and Flynn 2011). Even though activated macrophages can initialize phagocytosis, Mtb can also set up various strategies against macrophage killing and replicate within the host macrophages. Moreover, cytokine environment is also modified to favor the intracellular Mtb survival (BoseDasgupta and Pieters 2014; Orme et al. 2015). Innate immune responses are the crucial factor for the TB outcome (Orme et al. 2015). While the clearance of the phagocytized bacteria is also determined by the adaptive immunity, which is activated by innate immune signals (Torrado and Cooper 2013). These infected macrophages release chemotactic signals to recruit and activate migrating lymphocytes to the site of infection (Gammack et al. 2005; Gideon and Flynn 2011). The progressive immune responses lead to an aggregation of lymphocytes around mycobacteria-harboring macrophages, which is called a granuloma. Granulomas are able to isolate infected macrophages from the rest of the lung to limit the bacillary growth and spread of Mtb from intracellular Mtb replication, and so serve as an immune micro-environment to enhance the $\mathrm{T}$ cells and macrophages interactions (Russell et al. 2010; Gideon and Flynn 2011). On the other hand, the granuloma concealed Mtb switch to a non-replicating state, which is a typical feature to enable the bacillus persistence during the long TB latency period (Barry et al. 2009). Mtb has evolved new strategy to escape granulomas: leak into the lung and expectorate bacilli (Ehlers and Schaible 2013). The granuloma then serves as a battle ground between mycobacteria and host immune cells. The host-pathogen dynamics decide granuloma to promote or inhibit the infection and then determine the disease outcomes (Ulrichs and Kaufmann 2006; Ehlers and Schaible 2013). An imbalanced interplay between 
Mtb and immune cells result in the failure of controlling infection, then lead to the LTBI reactivation (O' Garra et al. 2013).

Today, TB disease can be cured with antibiotics. For the newly diagnosed pulmonary TB, four first-line anti-TB drugs isoniazid (INH), rifampin (RIF), ethambutol (EMB), and pyrazinamide (PZA) form the essence of treatment regimens (Organization 2017). The major problem of the current TB drug regimen is its duration, which requires 6 to 9 months. Incorrect TB drug treatment (the wrong length of duration or dose) could lead the remaining alive Mtb resistant to those drugs. These patients are more likely to develop drug-resistant TB, which is more difficult to treat. The goal of shortening the long treatment duration can advance treatment effectiveness by improving patients' compliance and reducing the chance of the drug resistance and disease relapse. Mathematical models may be useful tools for, first, elucidating in-host TB progression mechanisms, and then also for suggesting crucial factors for advancing therapeutic strategies.

\subsection{Mathematical modeling background}

Numerous TB in-host modeling studies have been done to identify the driving factor for different TB infection outcomes. The TB host-immune modeling began with models considering HIV-1 and Mtb coinfection by Kirschner (1999) and her collaborators Bauer et al. (2008). HIV infection weakens the immune system, and make patients vulnerable to opportunistic infections, such as TB. Untreated latent TB and HIV coinfection significantly increases the possibility of developing TB disease. The study of TB infection is carried out through ODE models to capture the cellular and cytokine network (Antia et al. 1996; Abdelrazec et al. 2016; Wigginton and Kirschner 2001; Magombedze et al. 2006) and CD8+ T cells for host infection control (Sud et al. 2006; Magombedze et al. 2006), PDE models have been developed for cytokine and antibiotics molecules diffusion (Lauffenburger and Linderman 1993), agent-based models for spatial and temporal features of granuloma (Segovia-Juarez et al. 2004; Ray et al. 2009) and multiple scale models for investigating the granulomas dynamics in a smaller scale (such as a lung) and a larger scale (such as a host body) (Gong et al. 2015; Marino and Kirschner 2016). However, little work has been done to investigate the determining factors for LTBI reactivation. One of the challenges is the computational cost due to the large number of considered compartments and parameters, combined with complex nonlinear terms. In this paper, we only consider the key factors involved in the in-host Mtb dynamics, which include the infection interaction and innate immune response between macrophages and bacteria, the adaptive immune response initiated by $\mathrm{T}$ cells, and bacterial growth and elimination processes.

A four-dimensional within-host TB model was developed in previous modeling work by Du et al. (2017). This 4-dimensional model, presented in (1), incorporates the uninfected macrophages (the Mtb target cells), infected macrophages, Mtb bacteria and CD4 T cells. The model numerically captures various TB infection outcomes, which are clearance, latency, and active disease. In an analytical study of the same model (Zhang et al. 2020), we showed the existence of four coexisting equilibria that 
reflect the different disease outcomes. We also determined conditions for forward and backward bifurcations (Zhang et al. 2020). However, neither (Du et al. 2017) or (Zhang et al. 2020) has analyzed the driving factors behind the different outcomes of disease.

\subsection{Overview}

We provide a brief immunology and mathematical modeling background of tuberculosis infection in Sect. 1. In Sect. 2, we introduce an established in-host tuberculosis model, then identify parameters statistically significantly influencing the early stage TB infection through a sample-based sensitivity analysis on the basic reproduction number. Using identified parameters from the first sensitivity analysis, we employ a second sensitivity analysis on the single-zero eigenvalue bifurcation condition and identify that macrophage apoptosis rate is the statistically significantly parameter for disease progression, especially LTBI reactivation. In Sect. 3, using bifurcation analysis and numerical simulations, various disease outcomes are fully unfolded under the variation of the macrophage apoptosis rate. In Sect. 4, we describe how the mathematical results from the previous section reveal the complicated roles of macrophage apoptosis in the complex Mtb-macrophage dynamics. In Sect. 5, we further investigate the disease outcomes under the influence of macrophage apoptosis and one of the other four parameters to give insight into the development of new therapeutic strategies. Our findings suggest that macrophage apoptosis modulation therapy can be enhanced with combination therapies, which inhibit bacillary viability and promote adaptive immunity.

\section{Investigation of the driving factors for disease progression through sensitivity analysis}

Tuberculosis progression is characterized by reactivation of LTBI, which occurs when a weakened immune system is no longer capable of controlling the latent stage Mtb. The awoken bacteria become active, defeat the immune defense, and lead to the infected individual to active TB disease. In other words, the LTBI reactivation can be described as the Mtb bacterial level shifted from a lower infection steady state to a higher infection steady state. Our target in this section is to identify the important factors determining the shifting of Mtb bacterial level by evaluating the influence/sensitivity level of each involving mechanism applied on the shift.

\subsection{Model}

The TB model with various disease outcomes (Du et al. 2017) is written as follows:

$$
\begin{aligned}
& \frac{d M_{u}}{d t}=s_{M}-\mu_{M} M_{u}-\beta M_{u} B \\
& \frac{d M_{i}}{d t}=\beta M_{u} B-b M_{i}-\gamma M_{i} \frac{T / M_{i}}{T / M_{i}+c}
\end{aligned}
$$




$$
\begin{aligned}
& \frac{d B}{d t}=\delta B\left(1-\frac{B}{K}\right)+M_{i}\left(N_{1} b+N_{2} \gamma \frac{T / M_{i}}{T / M_{i}+c}\right)-M_{u} B\left(\eta+N_{3} \beta\right) \\
& \frac{d T}{d t}=s_{T}+\frac{c_{M} M_{i} T}{e_{M} T+1}+\frac{c_{B} B T}{e_{B} T+1}-\mu_{T} T .
\end{aligned}
$$

Here, uninfected macrophages $M_{u}$ are generated and die naturally at constant rates $s_{M}$ and $\mu_{M}$. Moreover, they become infected through the engulfment of Mtb at a rate of $\beta M_{u} B$. The internalized Mtb reproduction can kill infected macrophages at a rate $b$ and produce $N_{1}$ intracellular Mtb bacteria per cell, on average. Infected macrophages can also be eliminated through $\mathrm{T}$ cell-mediated cytotoxicity. Both CD4 and CD8 $\mathrm{T}$ cells account for the cytotoxic process. The rate of the cytotoxic effect, which $\mathrm{T}$ lymphocytes (E) act on Mtb-stimulated monocytes (T), depends on the E:T ratio, $T / M_{i}$ (Lewinsohn et al. 1998; Wigginton and Kirschner 2001). This ratio has a maximum rate $\gamma$ and saturating factor $c$. During the cytotoxic process, infected macrophages die and release on average $N_{2}$ intracellular Mtb bacteria to the extracellular compartment $B$. Extracellular bacteria can divide, modeled as a logistic term $B(1-B / K)$. The infection in macrophages can lead to a loss of extracellular Mtb. This is modeled using the term $N_{3} \beta M_{u} B$. On the other hand, uninfected macrophages phogocytize Mtb bacteria, then initiate phagosome maturation, which causes microbicidal and degrading effects on phogocytized pathogens. The Mtb killing rate by this process is denoted as $\eta B M_{u}$ (Kinchen and Ravichandran 2008; Upadhyay et al. 2018). Host adaptive immune responses are signaled by CD4 T cells, which are assumed to have constant production and death rates $s_{T}$ and $\mu_{T}$. CD4 T cells can proliferate in the presence of Mtb bacteria $\left(\frac{c_{B} B T}{e_{B} T+1}\right)$ or/and infected macrophages $\left(\frac{c_{M} M_{i} T}{e_{M} T+1}\right)$. More detailed model assumptions are explained in Du et al. (2017) and parameters values are stored in Table 1.

\subsection{Basic model properties}

A detailed analytical investigation on the property of model (1) is carried out in Zhang et al. (2020). For convenience, we change the notation as

$$
\begin{gathered}
y_{1}=M_{u}, y_{2}=M_{i}, y_{3}=B, y_{4}=T \\
f_{1}=\frac{d M_{u}}{d t}, f_{2}=\frac{d M_{i}}{d t}, f_{3}=\frac{d B}{d t}, f_{4}=\frac{d T}{d t} .
\end{gathered}
$$

The solutions of model (1) are well-posed and bounded and include a disease free equilibrium $\left(E_{0}\right)$ and infected equilibriums $\left(E^{*}\right)$ as follows (Zhang et al. 2020) 


$$
\begin{aligned}
E_{0}= & \left(\frac{s_{M}}{\mu_{M}}, 0,0, \frac{s_{T}}{\mu_{T}}\right), \quad \text { and } E^{*}=\left(\bar{y}_{1}, \bar{y}_{2}, \bar{y}_{3}, \bar{y}_{4}\right), \quad \text { where, } \\
\bar{y}_{1}\left(y_{3}\right)= & \frac{s_{M}}{\beta y_{3}+\mu_{M}}, \\
\bar{y}_{2}\left(y_{3}\right)= & \left(\frac{y_{3} \delta}{K}-\frac{\delta+s_{M}\left(N_{2}-N_{3}\right) \beta-s_{M} \eta}{\beta y_{3}+\mu_{M}}\right) \frac{y_{3}}{b(N 1-N 2)}, \\
& \quad \text { if } \frac{y_{3} \delta}{K}>\frac{\delta+s_{M}\left(N_{2}-N_{3}\right) \beta-s_{M} \eta}{\beta y_{3}+\mu_{M}} \text { and } N_{1}>N_{2}, \\
\bar{y}_{4}\left(y_{3}\right)= & \frac{\left[\beta s_{M} y_{3}-\left(\beta y_{3}+\mu_{M}\right) b y_{2}\right] c y_{2}}{\beta s_{M} y_{3}-(b+\gamma)\left(\beta y_{3}+\mu_{M}\right) y_{2}}, \quad \text { and } \\
F\left(y_{3}\right)= & -e_{B} e_{M} \mu_{T} \bar{y}_{4}^{3}+\left[\left(c_{M} \bar{y}_{2}+e_{M} s_{T}-\mu_{T}\right) e_{B}+e_{M}\left(c_{B} y_{3}-\mu_{T}\right)\right] \bar{y}_{4}^{2} \\
& +\left[c_{B} y_{3}+c_{M} \bar{y}_{2}\left(y_{3}\right)+e_{B} s_{T}+e_{M} s_{T}-\mu_{T}\right] \bar{y}_{4}\left(y_{3}\right)+s_{T}=0 .
\end{aligned}
$$

Furthermore, equilibrium solutions $E_{0}$ and $E_{*}$ intersect and exchange local stability at a transcritical bifurcation as

$$
\beta=\frac{\left(-\delta \mu_{M}+\eta s_{M}\right)(b+\gamma)}{s_{M} \gamma\left(N_{2}-N_{3}\right) \gamma+s_{M} b\left(N_{1}-N_{3}\right)}:=\beta_{T} .
$$

Moreover, at the transcritical bifurcation the basic reproduction number equals to unit (Du et al. 2017), i.e. $R_{0}=1$, where

$$
\begin{aligned}
R_{0} & =\frac{\left[\gamma\left(N_{2}-N_{3}\right)+b\left(N_{1}-N_{3}\right)\right] \beta}{\left(-\delta \mu_{M} / s_{M}+\eta\right)(b+\gamma)} \\
& =\left[\left(N_{2}-N_{3}\right)\left(1-\frac{b}{b+\gamma}\right)+\left(N_{1}-N_{3}\right) \frac{b}{b+\gamma}\right] \frac{\beta}{\eta-\frac{\mu_{M}}{s_{M}} \delta} .
\end{aligned}
$$

\subsection{Sensitivity analysis of $\boldsymbol{R}_{0}$ for the early stage of TB infection}

After the exposure of TB disease, the critical value to determine the fate of the infection is the basic reproduction number $R_{0}$. Starting from one infectious pathogen, the infection will be able to spread to the whole body if $R_{0}>1$, but will be eliminated if $R_{0}<1$. Because $R_{0}$ is not a constant number for any given disease, in the case of in-host TB dynamics, $R_{0}$ is affected by in-host immunological factors and Mtb behaviors. We first investigate $R_{0}$ in (5) for all model parameters in the experimental and/or estimated ranges from Table 1 . We then choose $R_{0}$ in (5) as the first sensitivity/output function of the model parameters (or input factors) to identify which parameters significantly affect the uncertainty on disease spread and elimination in the early stage of the TB infection. The analytical formulation (5) indicates that $R_{0}$ is linear on the parameters $\beta, N_{1}, N_{2}$, and $N_{3}$, along with the ratio $\frac{b}{b+\gamma} \cdot R_{0}$ also depends on the term $\frac{\mu_{M}}{s_{M}} \delta$. 


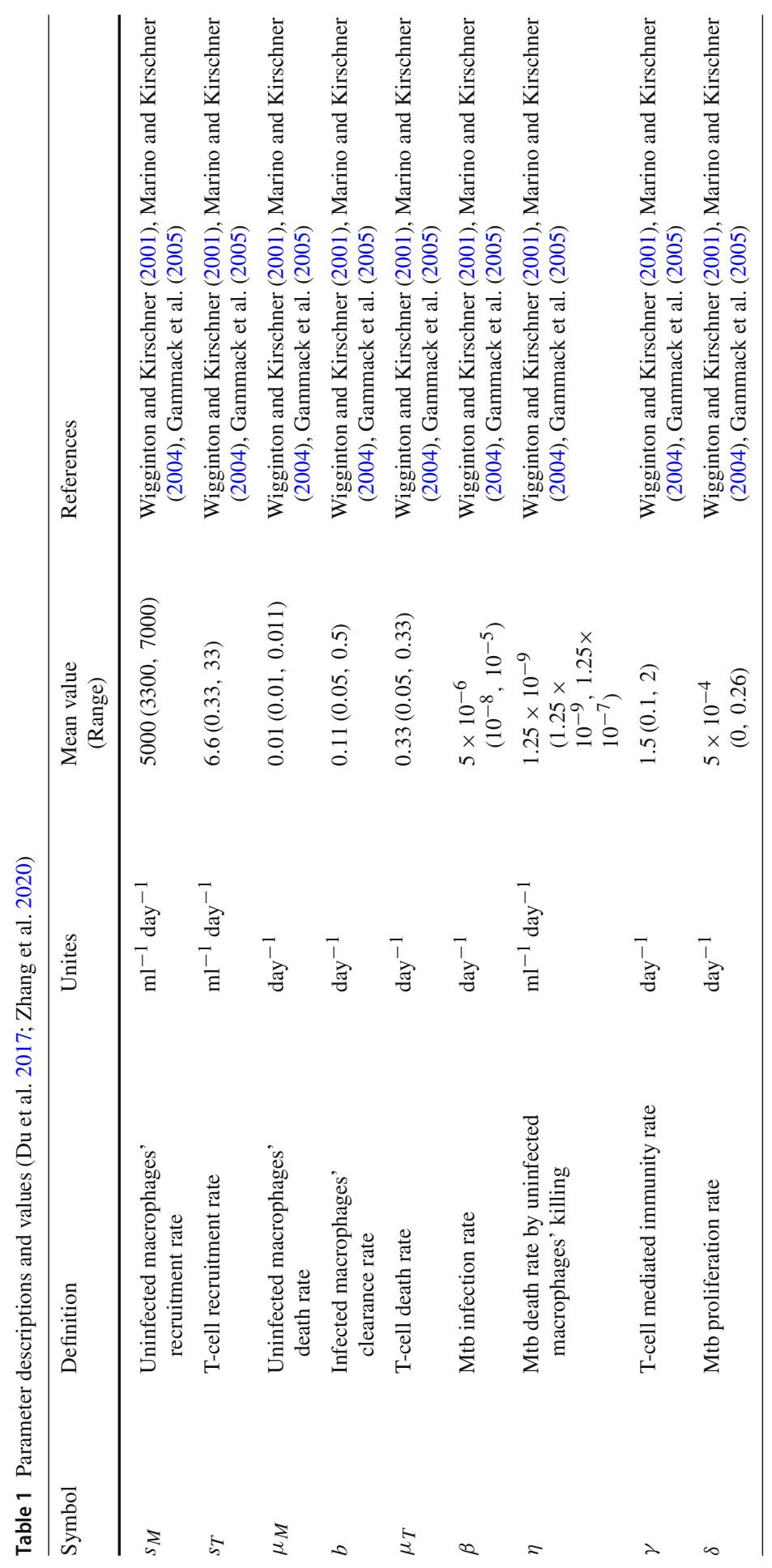




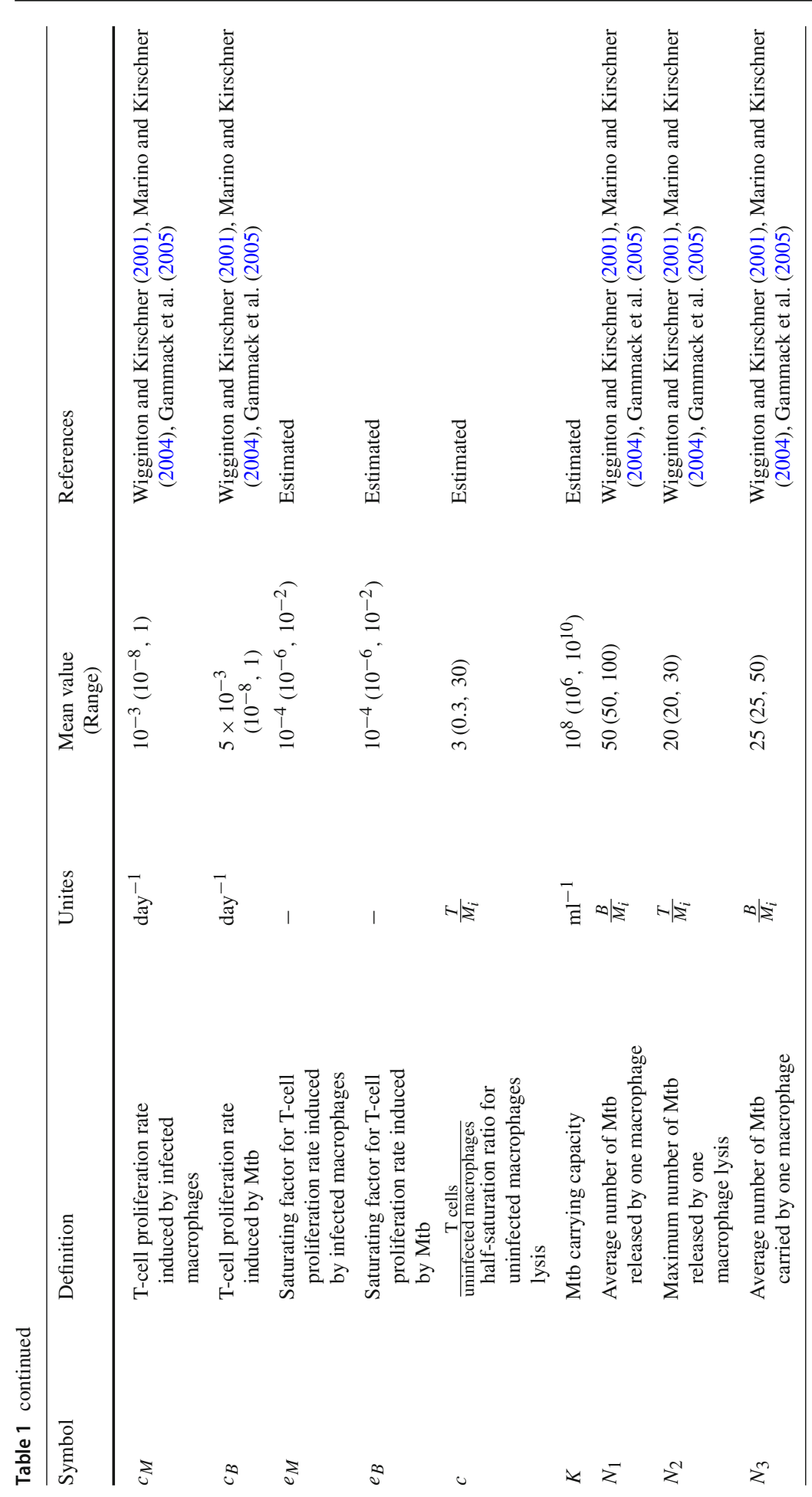


To show the uncertainty on $R_{0}$ due to the uncertainties in model parameters, we perform uncertainty analysis. Because a priori knowledge of the Mtb-host relationship between the model parameter and $R_{0}$ is unavailable, we choose the most popular sampling-based approach, i.e. Latin Hypercube sampling (LHS) (McKay et al. 1979). LHS belongs to the Monte Carlo class of sampling procedures for the propagation of uncertainty. LHS performs a full stratification over each sampled-parameter range to allow every parameter get equally sampled, and returns unbiased estimates for the sample mean and distribution function over each sampled-parameter range. Moreover, when necessary, a log scale can be applied on the parameter range for LHS to prevent under-sampling (McKay et al. 1979; Marino et al. 2008). Furthermore, LHS nearly eliminates confounding in estimating parameter effects by ensuring that exactly one parameter is sampled from each stratum in a given sample. That is, if there are 3 parameters, then each parameter range is partitioned into 3 strata and one parameter is sampled from the lowest stratum, another from the middle stratum, and the last from the highest stratum. This is repeated many times to ensure that all parameters are sampled from each of their sampling strata. This results in the main effects of each parameter being confounded only with high-order interactions of other parameters, which are classically considered negligible in experimental design. This also makes LHS a computationally inexpensive approach, especially compared to full factorial sampling designs, and allows LHS to perform robustly for even a relatively small sample size, i.e. sample size $L H S=50$ to 200, which is used in Iman and Helton (1988); Kleijnen and Helton (1999); Helton and Davis (2003). As such, this sampling design facilitates a measurement of parameter's corresponding impact on the value of the sensitivity functions $R_{0}$ in (5) and $a_{4}\left(E^{*}, p_{v}\right)$ in (12).

Due to the multi-variable property of sensitivity functions, standard correlation coefficients (CCs) are not sufficient for measuring the influence of parameter values on sensitivity functions because CCs are restricted to quantify relationships between just two variables and, as such, cannot account for the impact of the other parameters on the variation in sensitivity functions. Instead, we can calculate partial correlation coefficients (PCCs), which quantify the relationship between each parameter on the portion of the variation in sensitivity functions that is unexplained by the variation in the values of the other parameters and thusly, provide a more appropriate measure of influence. However, PCCs only reflect the linear relationships between the output of sensitivity functions and the input variables/parameters. To capture the nonlinear relationships between sensitivity functions and the model parameters, we can instead apply the rank transformation to all quantities (Conover and Iman 1981; Saltelli and Sobol 1995). Calculating the correlations between these provided us with the Spearman correlation coefficients, which measure the strength and direction of monotonic relationships. PCCs for the ranked quantities yields partial rank correlation coefficients (PRCC), which provide a robust sensitivity measure for quantifying potentially nonlinear, but monotonic, relationships between the output and each of the model parameters in the presence of the other parameters (Marino et al. 2008; Saltelli and Annoni 2011).

Using the MATLAB LHS and PRCC code provided in Marino et al. (2008), we run an LHS uncertainty analysis on a sample of 1000 parameter sets, and set the baseline value/mean, lower bound, and upper bound for each parameter in its range from Table 


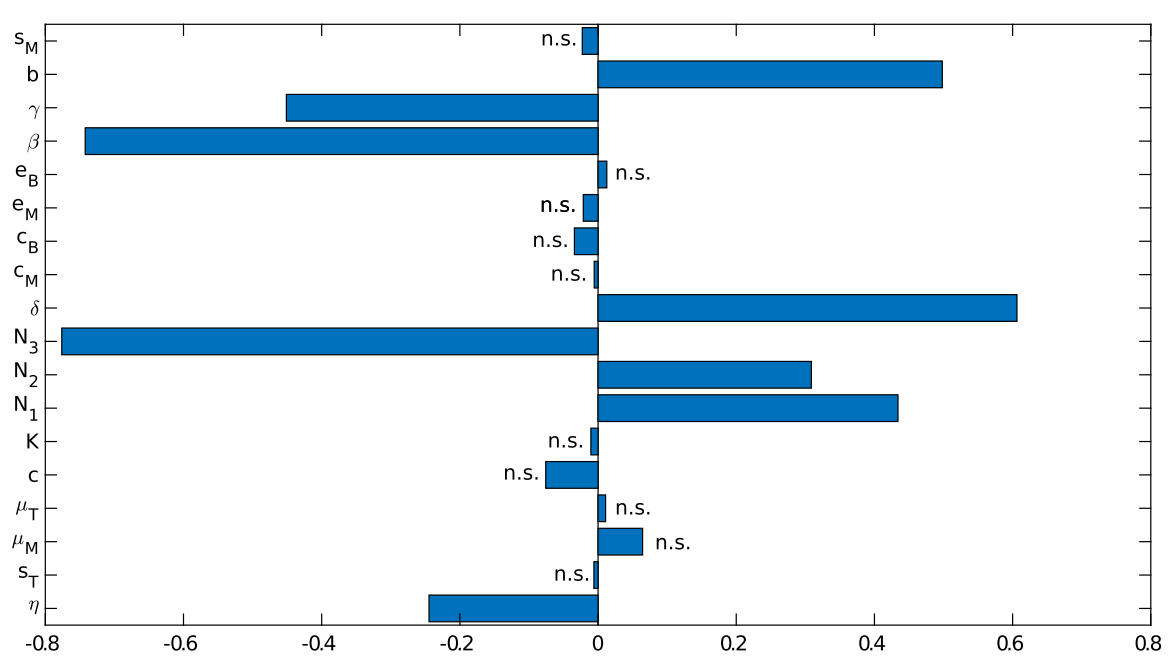

Fig. 1 PRCC values for $R_{0}$ under the variations of all model parameters. n.s. denotes statistically not significant, that is $p$ value $>0.05$

1. Scatter plots showing the relationships between residuals of the output $R_{0}$ and input parameters, along with their PRCCs and associated p-values, are shown in Figure 1 in Appendix (see supplementary material). From these figures, it is readily apparent that all of the pairs with near-zero PRCCs display no evidence of non-monotonic relationships, so PRCCs appropriately describe the relationships. A summary of the PRCC values is shown as a bar graph in Fig. 1. Following traditional use, p-values below 0.05 designate pairs of parameters whose partial rank correlations are statistically significantly different than 0 . The negative or positive sign of PRCC indicate the negative or positive correlation between $R_{0}$ and the specific parameter.

The PRCC between $R_{0}$ and each parameters in Fig. 1a indicate that the expected number of bacteria, $R_{0}$, generated by one single bacterium after exposure to Mtb has

- a significantly positive relation with $N_{1}, N_{2}$, and $N_{3}$;

- a significantly negative relation with $\beta$ because the infection process involves the loss of extracellular Mtb by macrophages phagocytosis;

- a significantly negative relation with $\eta$;

- a significant relation with the whole term $\frac{b}{b+\gamma}$, indicating that more intracellular Mtb are released from apoptosis than necrosis (Lee et al. 2009);

- a positive relation with the whole term $\frac{\mu_{M}}{s_{M}} \delta$.

There is an identifiablility issue with the parameters $\delta, s_{M}$ and $\mu_{M}$, because they only appear in (5) together as the term $\frac{\mu_{M}}{s_{M}} \delta$. While each of these parameters should be expected to have a non-zero rank correlation with $R_{0}$, since partial correlation accounts for the effects of the other parameters, only one of these three can have a high PRCC value. In this case, the effect appeared present for $\delta$ rather than the other two. Since the uninfected macrophages level in a healthy host is relatively constant, the Mtb proliferation rate $\delta$ is chosen to represent the significantly positive influence on $R_{0}$. 
Also note that the other non-influential parameters are $s_{T}, \mu_{T}, c, K, c_{M}, c_{B}, e_{M}, e_{B}$, which do not show in the analytical formula of $R_{0}$ in (5).

\subsection{Sensitivity analysis of saddle-node bifurcations for LTBI reactivation}

LTBI reactivation is characterized by an in-host Mtb outbreak. The Mtb level shifts from a low-level latent infection stage to a high-level active disease stage by the slowly weakening immune system, which may be caused by AIDS, chemotherapy, aging, and diabetes. In our mathematical model, the LTBI reactivation can be captured by the bacteria level traveling from a low steady state to a high steady state due to slowly changing parameter values. The prerequisite condition is the existence of co-existing steady states. The fundamental mechanism for the generation and destruction of steady states is the occurrence of saddle-node bifurcation. Therefore, the parameter influence on the shifting of the infection steady states is replaced by the parameter influence on the occurrence of saddle-node bifurcation. The sensitivity analysis of the LTBI reactivation is taken over by the sensitivity analysis of the saddle-node bifurcation conditions.

In general, we consider an $n$-dimensional nonlinear system

$$
\frac{d x}{d t}=f(x, p), \quad x \in R^{n}, \quad p \in R^{m}, \quad f: R^{n+m} \rightarrow R^{n},
$$

with $m$ parameter values and equilibrium solutions $x_{e}=x_{e}(p)$ derived from the equilibrium condition

$$
f\left(x_{e}(p), p\right)=0, \quad x \in R^{n}, \quad p \in R^{m} .
$$

The local stability of the equilibrium points $x_{e}(p)$ is determined by the eigenvalues of the Jacobian $J(p)=\left[\partial f_{i}\left(x_{e}(p), p\right) / \partial x_{j}\right]$, which are the roots of the corresponding characteristic polynomial equation

$$
P_{n}(\lambda)=\operatorname{det}[\lambda I-J(p)]=\lambda+a_{1}(p) \lambda^{n-1}+a_{2}(p) \lambda^{n-2}+\cdots+a_{n-1}(p) \lambda+a_{n}(p) .
$$

The necessary and sufficient conditions for zero singularities are given in Yu (2005).

Theorem $1 \mathrm{Yu}$ (2005) The necessary and sufficient conditions for system (6) to have a k-zero singularity at a fixed point (equilibrium), $x=x_{e}(p)$, of the system are given by

$$
a_{n}(p)=a_{n-1}(p)=\cdots=a_{n+1-k}(p)=0,
$$

which $a_{i}(p)$ 's are the coefficients of the characteristic polynomial (8). Further, if the remaining coefficients $a_{1}, a_{2}, \ldots a_{n-k}$ still obey the Hurwitz conditions for order $n-k$, then all the remaining eigenvalues of the Jacobian have negative real parts.

For the current case, we only need to study single zero bifurcations, that is $k=1$. The corresponding necessary condition becomes $a_{4}(p)=0$, since the dimension of model (1) is 4 . Single zero bifurcations include saddle-node/turning bifurcation, 
Fig. 2 PRCC values for single zero-eigenvalue bifurcations $a\left(y_{3}, p_{v}\right)$ in (12) on the influential parameters for disease outcomes $p_{v}$

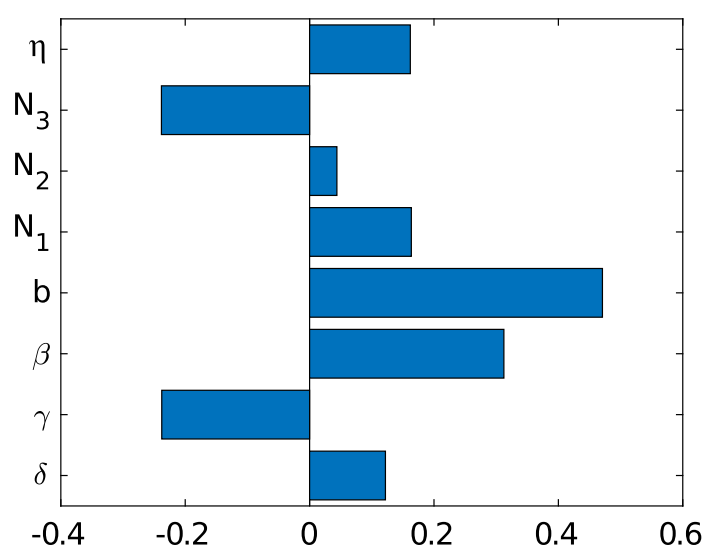

which creates or destroys steady states, transcritical bifurcation (at $R_{0}=1$ ), which serves as the intersection between infection-free and infected steady states (Zhang et al. 2020), and pitchfork bifurcation, which is not common in within-host infectious disease model, due to the symmetric property.

The sensitivity function on LTBI reaction for model (1) is represented by the sensitivity function of the necessary condition of single zero bifurcation (denoted by $a_{4}(p)=0$ ) in terms of the parameters (denoted by $p_{v}$ ) significantly affecting the transcritical bifurcation condition $\left(R_{0}=1\right)$. The sensitivity analysis in Sect. 2.3 identified the significant parameters affecting $R_{0}$ to be

$$
p_{v}=\left(b, \gamma, \beta, \delta, \eta, N_{1}, N_{2}, N_{3}\right)
$$

We fix the parameters, which are not significantly affecting $R_{0}$ or the fate of disease as the corresponding mean values in Table 1 . There parameters are denoted as

$$
p_{f}=\left(s_{T}, s_{M} \mu_{M}, \mu_{T}, c, K, c_{M}, c_{B}, e_{M}, e_{B}\right) .
$$

Due to the uncertainties of the input parameters $p_{v}$, we run Latin Hypercube sampling for 10, 000 sampled parameter set. The sensitivity function/output is set as

$$
a_{4}\left(E^{*}, p_{v}\right)=a_{4}\left(y_{3}, b, \gamma, \beta, \delta, \eta, N_{1}, N_{2}, N_{3}\right),
$$

where $E^{*}$ denotes the infected steady state and $y_{3}$ satisfies $F\left(y_{3}\right)=0$ in (3). Moreover, the non-influential parameters $p_{f}$ in terms of disease outcomes are fixed. Scatter plots showing the relationships between the disease-outcome influential parameters $p_{v}$ and the sensitivity function $a_{4}\left(E^{*}, p_{v}\right)$ in (12) are shown in Fig. 2. As in the preceding sensitivity analysis, these plots show no evidence of non-monotonicity, which again suggests that PRCC scores are appropriate to use. The PRCC scores for these are shown in bar graph form in Fig. 2. The sensitivity analysis identifies that

- $b$ has the largest magnitude PRCC value with the sensitivity function $a_{4}\left(E^{*}, p_{v}\right)$;

- $N_{2}$ is non-influential to the LTBI reactivation; 
- the other parameters in $p_{v}$ are statistically significant but have a moderate absolute PRCC value with the sensitivity function/output $a_{4}\left(E^{*}, p_{v}\right)$.

To further investigate how the identified significant parameter affects disease progression and what disease outcome will show up, we set $b$ as a bifurcation parameter of interest for a one-parameter bifurcation analysis in the next section. More twodimensional bifurcation diagrams are provided in the later section on the identified influential parameters to provide insight on TB treatment strategies.

\section{Bifurcation analysis of the macrophages programmed cell death rate}

\subsection{Investigation of the single-zero eigenvalue bifurcation}

The single-zero eigenvalue bifurcations are derived according to Theorem 1. Leaving the most influential parameter $b$ as the bifurcation parameter, fixing the other influential parameters in $p_{v}$ and non-influential parameters in $p_{f}$ as the corresponding mean value in Table 1, we rewrite model (1) using the new notation in (2) as

$$
\dot{y}=f(y)=\left[f_{1}(y, b), f_{2}(y, b), f_{3}(y, b), f_{4}(y, b)\right]^{T}, \quad y=\left(y_{1}, y_{2}, y_{3}, y_{4}\right) \in \mathbb{R}^{4} .
$$

Suppose the steady state/equilibrium is given in the form

$$
\bar{y}=\bar{y}(b)=\left(\bar{y}_{1}(b), \bar{y}_{2}(b), \bar{y}_{3}(b), \bar{y}_{4}(b)\right),
$$

which could represent multiple co-existing equilibrium solutions. Then the Jacobian matrix of (13) at the infected equilibrium $E^{*}$ in (3) is

$$
J(b)=\left.D_{y} f\right|_{y=\bar{y}(b)}=\frac{\partial f_{i}(\bar{y}(b), b)}{\partial y_{j}},
$$

where $\bar{y}(b)$ satisfies the equilibrium conditions in (3). We denote the fourth-degree characteristic polynomial in terms of $L$ at the infected equilibrium $E^{*}$ in (3) as

$$
P_{4}\left(L ; y_{3}, b\right)=L^{4}+a_{1}\left(y_{3}, b\right) L^{3}+a_{2}\left(y_{3}, b\right) L^{2}+a_{3}\left(y_{3}, b\right) L+a_{4}\left(y_{3}, b\right),
$$

where $y_{3}$ satisfies the equilibrium condition in (3) $F\left(y_{3}, b\right)=0$. Evaluating this with the parameter values in Table 1 , the formula of $F\left(y_{3}, b\right)$ and $a_{i}\left(y_{3}, b\right) i=1, \ldots, 4$ are shown in Appendix (see supplementary material) Equations (1),(3),(4),(5), and (6). We assume

(SN1) the Jacobian matrix $J(b)=\left.D_{y} f\right|_{y=\bar{y}(b)}=D_{y} f\left(E^{*}, b\right)$ has a simple zero eigenvalue, at $b=b_{T}$, if (1) $\Delta_{1}=a_{1}\left(y_{3}, b\right)>0$, (2) $\Delta_{2}=$ $a_{1}\left(y_{3}, b\right) a_{2}\left(y_{3}, b\right)-a_{3}\left(y_{3}, b\right)>0$, (3) $\Delta_{3}=\left[a_{1}\left(y_{3}, b\right) a_{2}\left(y_{3}, b\right)-\right.$ $\left.a_{3}\left(y_{3}, b\right)\right] a_{3}\left(y_{3}, b\right)-a_{1}^{2}\left(y_{3}, b\right) a_{4}\left(y_{3}, b\right)>0$, and (4) $\Delta_{4}=\Delta_{3} a_{4}\left(y_{3}, b\right)=$ 0 or $a_{4}\left(y_{3}, b\right)=0$. Then $b=b_{T}$ and $E^{*}=E^{*}\left(b_{T}\right)$ is called critical point (Yu 2005). 
We obtain four single-zero bifurcation critical points:

Single-zero 1. $b_{T 1}=0.0295$ day $^{-1}, E_{1}^{*}=(497833,122,8.7,40)$ cells $/ \mathrm{ml}$.

Single-zero 2. $b_{T 2}=0.2993 \mathrm{day}^{-1}, E_{2}^{*}=(21089,2664,45417,6952168)$ cells $/ \mathrm{ml}$.

Single-zero 3. $b_{T 3}=0.1363 \mathrm{day}^{-1}, E_{3}^{*}=(20,3055,49998972,7575684467)$ cells $/ \mathrm{ml}$.

Single-zero 4. $b_{T 4}=0.3000036 \mathrm{day}^{-1}, E_{4}^{*}=(500000,0,0,20) \mathrm{cells} / \mathrm{ml}$.

Single-zero bifurcation include saddle-node/turning bifurcation, transcritical bifurcation, and pitchfork bifurcation, through the pitchfork bifurcation is not common in infectious disease models due to the symmetric pattern. To identify the type of the single-zero bifurcation and the local behavior of the corresponding vector field, We apply Theorem 3.4.1 in Guckenheimer and Holmes (1990). That is, for the system (13), there are three hypotheses SN1,

(SN2) $v \frac{\partial f}{\partial b}\left(E^{*}, b_{T}\right) \neq 0$

(SN2') $v\left(\frac{\partial f^{2}}{\partial b \partial y}\left(E^{*}, b_{T}\right)(w)\right) \neq 0$

(SN3) $v\left[D_{y}^{2} f\left(E^{*}, b_{T}\right)(w, w)\right] \neq 0$,

where $w$ and $v$ are the left and right eigenvectors corresponding to the 0 eigenvalue of the matrix $D_{y} f\left(E^{*}, b_{T}\right)=\left.D_{y} f\right|_{y=\bar{y}\left(b_{T}\right)}:=\left.D_{y} f_{b}(y)\right|_{y=\bar{y}\left(b_{T}\right)}$. Moreover the transversality and degeneracy conditions are defined respectively as

$$
\begin{gathered}
v\left[\frac{\partial f}{\partial b}\right]=v\left[\frac{\partial f_{1}}{\partial b}, \frac{\partial f_{2}}{\partial b}, \frac{\partial f_{3}}{\partial b}, \frac{\partial f_{4}}{\partial b}\right]^{T} \\
v\left[D_{y}^{2} f_{b}(w, w)\right]=v\left[w^{T}\left(\frac{\partial^{2} f_{1}}{\partial y_{j} \partial y_{k}}\right) w, \ldots, w^{T}\left(\frac{\partial^{2} f_{4}}{\partial y_{j} \partial y_{k}}\right) w\right]^{T} .
\end{gathered}
$$

If system (13) satisfies three hypotheses SN1, SN2, and SN3, there are no equilibriums that exist near $\left(E^{*}, b_{T}\right)$ when $b<b_{T}\left(b>b_{T}\right)$ and two equilibria near $\left(E^{*}, b_{T}\right)$ when $b>b_{T}\left(b<b_{T}\right)$. It implies a saddle-node bifurcation. If we replace SN2 by SN2', then a transcritical bifurcation occurs.

For the critical point $\left(b_{T 1}, E_{1}^{*}\right)$, the left and right eigenvectors corresponding to the simple zero eigenvalue of $\left.D_{y} f_{b}(y)\right|_{\left(b_{T 1}, E_{1}^{*}\right)}$ are chosen respectively as

$$
\begin{gathered}
v_{T 1}=[0.0045,21.9549,0.8369,-12.8169] \\
w_{T 1}=[-0.9982,0.0564,0.00403,0.01845]^{T},
\end{gathered}
$$

where $<v_{T 1}, w_{T 1}>=1$. The transversality and degeneracy conditions are satisfied since

$$
\begin{aligned}
& \left.v_{T 1}\left[\frac{\partial f}{\partial b}\right]\right|_{\left(b_{T 1}, E_{1}^{*}\right)}=2434.1057 \neq 0 \text { and } \\
& \left.v_{T 1}\left[w_{T 1}^{T}\left(D_{y}^{2} f_{b}\right) w_{T 1}\right]\right|_{\left(b_{T 1}, E_{1}^{*}\right)}=-0.3579 \times 10^{-4} \neq 0 .
\end{aligned}
$$


The signs of the transversality and degeneracy conditions indicate that no equilibrium point near $\left(b_{T 1}, E_{1}^{*}\right)$ when $b<b_{T 1}$, but two equilibria are near $\left(b_{T 1}, E_{1}^{*}\right)$ when $b>b_{T 1}$. Choosing $b=0.0298$, we test the stability of the two bifurcation equilibria: $E_{1}^{*+}=(497620,133,9.6,44)$ (associated with four negative real-part eigenvalues $-62.3025,-0.0864,-0.0096+0.0009 i$, and $-0.0096-0.0009 i)$ and $E_{1}^{*-}=(498014,111,8,37)$ (associated with one positive and three negative eigenvalues $-62.3471,-0.1356,-0.0099$, and 0.0059$)$. Given these results, a saddle-node bifurcation occurs at $\left(b_{T 1}, E_{1}^{*}\right)$ shown in Fig. 3a,b as $\mathrm{LP}_{1}$. The bifurcating upper branch equilibrium is stable and the lower branch is unstable.

For the critical point $\left(b_{T 2}, E_{2}^{*}\right)$, the left and right eigenvectors corresponding to the simple zero eigenvalue of $\left.D_{y} f_{b}(y)\right|_{\left(b_{T 2}, E_{2}^{*}\right)}$ are chosen respectively as

$$
\begin{gathered}
v_{T 2}=[0.001291-3578.6696,-143.1454,0.001432] \\
w_{T 2}=[0.0029,-0.000016,-0.0066,-0.99997]^{T}
\end{gathered}
$$

where $<v_{T 2}, w_{T 2}>=1$. The transversality and degeneracy conditions are satisfied since

$$
\begin{aligned}
& \left.v_{T 2}\left[\frac{\partial f}{\partial b}\right]\right|_{\left(b_{T 2}, E_{2}^{*}\right)}=-9533936.0987 \neq 0 \text { and } \\
& \left.v_{T 2}\left[w_{T 2}^{T}\left(D_{y}^{2} f_{b}\right) w_{T 2}\right]\right|_{\left(b_{T 2}, E_{2}^{*}\right)}=-0.1244 \times 10^{-9} \neq 0 .
\end{aligned}
$$

The other three negative eigenvalues associated with $\left.D_{y} f_{b}(y)\right|_{\left(b_{T 2}, E_{2}^{*}\right)}$ are -4.5701 , -0.3294 , and -0.0987 . Since both the transversality and degeneracy conditions are positive, there is no equilibrium near $\left(b_{T 2}, E_{2}^{*}\right)$ when $b>b_{T 2}$, but there are two equilibria near $\left(b_{T 2}, E_{2}^{*}\right)$ when $b<b_{T 2}$. To test the stability of the bifurcating equilibria, we choose $b=0.29$ and obtain an unstable upper equilibrium $E_{2}^{*+}=(705,2789,1415628,214563623)$ (associated with four eigenvalues $-7.2051,-1.7611,-0.32998$, and 0.00048 ) and a stable lower equilibrium $E_{2}^{*-}=$ $(468905,179,133,15543)$ (associated with four eigenvalues $-60.3205,-0.1793$, $-0.0083+0.0026 i$, and $-0.0083-0.0026 i)$. There also exist an unstable positive equilibrium $E_{T 2 l}=(499959,0.2307,0.1606,20.0627)$ (associated with four eigenvalues $-64.1962,-0.3289,-0.01$, and 0.0094$)$ and a stable positive equilibrium $E_{T 2 h}=(10,2793,98580761,14936553571)$ (associated with four eigenvalues $-492.9151,-1.78999,-0.3299$, and -0.00048). The dynamics are summarized in Fig. 3a near the point $\mathrm{LP}_{2}$.

For the critical point $\left(b_{T 3}, E_{3}^{*}\right)$, The left and right eigenvectors corresponding to the simple zero eigenvalue of $\left.D_{y} f_{b}(y)\right|_{\left(b_{T 3}, E_{3}^{*}\right)}$ are chosen respectively as

$$
\begin{gathered}
v_{T 3}=\left[-0.1103,0.9929,0.04412,-0.2447 \times 10^{-12}\right] \\
w_{T 3}=\left[-0.9064 \times 10^{-5}, 0.5385 \times 10^{-7}, 22.6606,3433.4342\right]^{T},
\end{gathered}
$$


where $<v_{T 3}, w_{T 3}>=1$. The transversality and degeneracy conditions are satisfied since

$$
\begin{aligned}
& \left.v_{T 3}\left[\frac{\partial f}{\partial b}\right]\right|_{\left(b_{T 3}, E_{3}^{*}\right)}=3707.9009 \neq 0 \text { and } \\
& \left.v_{T 3}\left[w_{T 3}^{T}\left(D_{y}^{2} f_{b}\right) w_{T 3}\right]\right|_{\left(b_{T 3}, E_{3}^{*}\right)}=-0.2266 \times 10^{-9} \neq 0 .
\end{aligned}
$$

The rest of the three negative eigenvalues associated with $\left.f_{b}(y)\right|_{\left(b_{T 3}, E_{3}^{*}\right)}$ are -250 , -1.6363 , and -0.3299$)$. The signs of the transversality and degeneracy conditions indicate no equilibrium near $\left(b_{T 3}, E_{3}^{*}\right)$ when $b<b_{T 3}$, and two equilibria near $\left(b_{T 3}, E_{3}^{*}\right)$ when $b>b_{T 3}$. Choosing $b=0.163$ we test the stability of the bifurcating branches and observe that there are two equilibria near $\left(b_{T 3}, E_{3}^{*}\right)$, a stable $E_{3}^{*+}=(14,3006,70982833,10755055859)$ (associated with four eigenvalues $-354.9259,-1.6629,-0.3299$, and -0.0002$)$ and an unstable $E_{3}^{*-}=$ (34, 3006, 29015090, 4396306898) (associated with four eigenvalues -145.0898, $-1.6629,-0.3299$, and 0.0002$)$. The other two unstable positive equilibria located further away from $\left(b_{T 3}, E_{3}^{*}\right)$ are $E_{T 3 h}=(489397,108,43,386)$ (associated with four eigenvalues $-61.9168,-0.0102,0.0537+0.1342 i$, and $0.0537-0.1342 i)$ and $E_{T 3 l}=(499421,6,2,21)$ (associated with four eigenvalues $-63.1107,-0.0099$, -0.3064 , and 0.0709). The dynamics are summarized in Fig. 3 a near the point $\mathrm{LP}_{3}$.

At $\left(b_{T 4}, E_{T 4}\right)$, the disease free equilibrium $E_{0}$ and infected equilibrium $E^{*}$ intersect and exchange stability. The qualitative behavior of the model (1) at $\left(b_{T 4}, E_{T 4}\right)$ is determined by its corresponding center manifold governed by $\dot{c}=\mathscr{A} c^{2}+\mathscr{B} \phi c$. The formula of the coefficients $\mathscr{A}$ and $\mathscr{B}$ are derived in our previous work (Zhang et al. 2020). Using the parameter values in Table 1 , we have $\left.\mathscr{A}\right|_{\left(b_{T 4}, E_{T 4}\right)}=4.3403$ and $\left.\mathscr{B}\right|_{\left(b_{T 4}, E_{T 4}\right)}=24.9999$. Therefore, the model (1) undergoes a transcritical bifurcation at $\left(b_{T 4}, E_{T 4}\right)$. Furthermore it shows a backward bifurcation due to the positiveness of the coefficients $\mathscr{A}$ and $\mathscr{B}$ in the quadratic terms. The dynamics are summarized in Fig. 3b near the point BP.

Note that the magnitudes of transversality and degeneracy conditions depend on the normalization of the corresponding left and right eigenvalues $v_{T 1}$ and $w_{T 1}$ (or $v_{T 2}$ and $w_{T 2}$ and $v_{T 3}$ and $w_{T 3}$ ). While the signs of transversality and degeneracy conditions (which are related to the opening direction of the saddle node bifurcation) are invariant under the scaling of the left and right eigenvectors. Therefore, we choose the left and right eigenvectors such that their dot product equals to one. Furthermore, the signs of transversality and degeneracy conditions for $E_{1}^{*}, E_{2}^{*}$, and $E_{3}^{*}$ show that the opening direction of the saddle node bifurcation $\mathrm{LP}_{2}$ is different with $\mathrm{LP}_{1}$ and $\mathrm{LP}_{3}$ (see Fig. $3)$.

\subsection{Investigation of the Hopf bifurcations}

The necessary and sufficient conditions (Wm 1994; Yu 2005; Yu and Wang 2019) for Hopf bifurcation $E^{*}$ require

Condition Hopf 1. $\Delta_{3}\left(y_{3}, b\right)=0$,

Condition Hopf 2. $\Delta_{1,2}\left(y_{3}, b\right)>0$, 
Condition Hopf 3. $\operatorname{det}\left(J\left(y_{3}, b\right)\right)>0$ (positive determinant of the corresponding Jacobian matrix),

Condition Hopf 4. $\frac{d \Delta_{3}\left(y_{3}, b\right)}{d b}=\frac{\partial \Delta_{3}\left(y_{3}, b\right)}{\partial b}+\frac{\partial \Delta_{3}\left(y_{3}, b\right)}{\partial B} \frac{d y_{3}}{d b}=\frac{\partial \Delta_{3}\left(y_{3}, b\right)}{\partial b}-\frac{\partial \Delta_{3}\left(y_{3}, b\right)}{\partial y_{3}}$ $\frac{\partial F / \partial b}{\partial F / \partial y_{3}} \neq 0$.

Here, $\Delta_{i}\left(y_{3}, b\right), i=1, \ldots, 4$ are Hurwitz arguments and the function $F\left(y_{3}\right)=0$ is the equilibrium condition. The above four criteria yield two Hopf bifurcation points:

Hopf 1. $b_{H 1}=0.0419$ day $^{-1}, E_{H 1}=(495822.4505,166.2001,16.8509,80.9264)$ cells/ml, with $\left.\Delta_{1}\right|_{\left(E_{H 1}, b_{H 1}\right)}=62.1427,\left.\Delta_{2}\right|_{\left(E_{H 1}, b_{H 1}\right)}=38.7987$, and $\left.\frac{d \Delta_{3}(B, b)}{d b}\right|_{\left(E_{H 1}, b_{H 1}\right)}=-5907.7516$, and $\operatorname{det}\left(J\left(E_{H 1}, b_{H 1}\right)\right)=0.003699$. The corresponding eigenvalues are $(-62.1326,-0.0100,0.07697 i,-0.07697 i)$ with $i^{2}=-1$.

Hopf 2. $b_{H 2}=0.2611 \mathrm{day}^{-1}, E_{H 2}=(485669.6896,91.4745,59.0125,1841.8417)$ cells $/ \mathrm{ml}$, with $\left.\Delta_{1}\right|_{\left(E_{H 2}, b_{H 2}\right)}=62.1708$, and $\left.\Delta_{2}\right|_{\left(E_{H 2}, b_{H 2}\right)}=39.7038$, $\left.\frac{d \Delta_{3}(B, b)}{d b}\right|_{\left(E_{H 2}, b_{H 2}\right)}=5475.2113, \operatorname{det}\left(J\left(E_{H 2}, b_{H 2}\right)\right)=0.005743$. The corresponding eigenvalues are $(-0.1605,-0.01027,0.09483 i,-0.09483 i)$.

We further study the post-critical behavior of the Hopf bifurcations to determine the stability of the bifurcating limit cycles. The corresponding Hopf bifurcation normal forms up to third order terms are derived through center manifold and normal form reductions. Due to the high non-linearity and stiffness of the model equations, we employ an established Maple program (Yu 1998) for the model analysis.

\subsubsection{The Hopf bifurcation at $b_{H 1}$}

We first apply a parameter-dependent shift of coordinate on the equilibrium condition in Eq. 3, that is

$$
F\left(y_{3 \mid E_{H 1}}+\varepsilon, b\right)=0 .
$$

Solving the preceding equation obtains

$$
b(\varepsilon)=b_{H 1}+0.0023 \varepsilon+O\left(\varepsilon^{2}\right) .
$$

Taking only the linear approximation of $b(\varepsilon)$, we assume that $b(\varepsilon)=b_{H 1}+\mu$, where $\mu=0.0023$ varepsilon, $\mu$ and $\epsilon$ are small perturbation parameters. It follows that $E_{H 1}^{*}(\epsilon)=E_{H 1}+[-245.8399 \epsilon, 0.7032 \epsilon, \epsilon, 5.4797 \epsilon]^{T}+O\left(\epsilon^{2}\right)$. We introduce the transformation

$$
\left[\begin{array}{l}
y_{1} \\
y_{2} \\
y_{3} \\
y_{4}
\end{array}\right]=E_{H 1}^{*}(\epsilon)+T_{H 1}\left[\begin{array}{l}
u_{11} \\
u_{12} \\
u_{13} \\
u_{14}
\end{array}\right], \quad T_{H 1}=\left[\begin{array}{cccc}
0.9581 & 0 . & -0.0398 & -0.9999 \\
0.1293 & -0.1352 & 0.0399 & -0.0002 \\
-0.0039 & -0.0297 & -0.9984 & 0.00001 \\
-0.0789 & -0.2002 & 0.0064 & -0.0001
\end{array}\right],
$$

into (1) and obtain

$$
\frac{d u_{1 i}}{d t}=g_{1 i}\left(u_{11}, u_{12}, u_{13}, u_{14} ; \epsilon\right), \quad \text { for } i=1,2,3,4,
$$


where $g_{1 i}$ for $i=1 \ldots 4$ are stored in Appendix (see supplementary material) (7)-(10). The corresponding Jacobian matrix of equations evaluated at the equilibrium $u_{1 i}=0$, $i=1,2,3,4$ takes the Jordan canonical form as

$$
J_{H 1}=\left[\begin{array}{cccc}
0 & \omega_{10} & 0 & 0 \\
-\omega_{10} & 0 & 0 & 0 \\
0 & 0 & -62.1326 & 0 \\
0 & 0 & 0 & -0.01005
\end{array}\right]
$$

where $\omega_{10}=0.07697$. The corresponding Hopf bifurcation normal form up to third order term is

$$
\frac{d r_{1}}{d t_{1}}=r_{1}\left(v_{10} \epsilon+v_{11} r_{1}^{2}\right), \quad \frac{d \theta_{1}}{d t_{1}}=\omega_{10}+\tau_{10} \epsilon+\tau_{11} r_{1}^{2},
$$

where $v_{10}$ and $\tau_{10}$ are derived from linear analysis as follows

$$
\begin{aligned}
& v_{10}=\left.\frac{1}{2}\left(\frac{\partial^{2} g_{11}}{\partial u_{11} \partial \epsilon}+\frac{\partial^{2} g_{12}}{\partial u_{12} \partial \epsilon}\right)\right|_{u_{1 i}=0, \epsilon=0}=0.0046752 \\
& \tau_{10}=\left.\frac{1}{2}\left(\frac{\partial^{2} g_{11}}{\partial u_{12} \partial \epsilon}-\frac{\partial^{2} g_{12}}{\partial u_{11} \partial \epsilon}\right)\right|_{u_{1 i}=0, \epsilon=0}=0.0042939 .
\end{aligned}
$$

The first focus value $v_{11}$ and the coefficient $\tau_{11}$ are derived from the Maple program (Yu 1998) taking $\epsilon=0$ as input. We obtain

$$
v_{11}=-0.1195 \times 10^{-6}, \quad \tau_{11}=-0.1632 \times 10^{-7} .
$$

The trivial equilibrium $\bar{r}_{10}=0$ indicates the equilibrium $E_{H 1}$ for model (1). While the nontrivial equilibrium $\bar{r}_{11}=-\frac{v_{10} \epsilon}{v_{11}}$ exists if $\epsilon>0$ and represents the amplitude of the bifurcating limit cycle. Since

$$
\left.\frac{d\left(v_{10} \epsilon r_{1}+v_{11} r_{1}^{3}\right)}{d r_{1}}\right|_{\bar{r}_{1}}=\left\{\begin{array}{r}
v_{10} \epsilon, \bar{r}_{1}=\bar{r}_{10} \\
-2 v_{10} \epsilon, \bar{r}_{1}=\bar{r}_{11}
\end{array}\right.
$$

The trivial equilibrium $\bar{r}_{10}=0$ is stable when $\epsilon<0$. When $\epsilon>0, \bar{r}_{10}=0$ becomes unstable, then a stable limit cycle merged with an amplitude of $\bar{r}_{11}$.

\subsubsection{The Hopf bifurcation at $b_{H 2}$}

Employing the similar approach as shown in the previous subsection, we first take the linear approximation of $b(\epsilon)$ solved from $F\left(y_{3 \mid E_{H 2}}+\epsilon, b\right)=0$. That is, $b(\epsilon)=$ 
$b_{H 2}+\mu$ with $\mu=0.0027 \epsilon$ ( $\mu$ and $\epsilon$ are small perturbations). Then we introduce the following transformation

$$
\begin{gathered}
{\left[\begin{array}{l}
y_{1} \\
y_{2} \\
y_{3} \\
y_{4}
\end{array}\right]=E_{H 2}^{*}(\epsilon)+T_{H 2}\left[\begin{array}{l}
u_{21} \\
u_{22} \\
u_{23} \\
u_{24}
\end{array}\right], \quad T_{H 1}=\left[\begin{array}{cccc}
0.2376 & -0.1206 & 0.0387 & -0.9999 \\
-0.0051 & -0.0149 & -0.0396 & -0.0002 \\
-0.0057 & -0.0087 & 0.9908 & 0.8749 \times 10^{-5} \\
-0.9637 & 0 . & -0.1231 & -0.0045
\end{array}\right],} \\
E_{H 2}^{*}(\epsilon)=E_{H 2}+[-235.8751 \epsilon, 0.5539 \epsilon, \epsilon, 158.9314 \epsilon]^{T}+O\left(\epsilon^{2}\right),
\end{gathered}
$$

into (1). It yields $\frac{d u_{2 i}}{d t}=g_{2 i}\left(u_{21}, u_{22}, u_{23}, u_{24} ; \epsilon\right)$, for $i=1,2,3,4$, where $g_{2 i}$ are stored in (11)-(14) in the Appendix (see supplementary material). The expression of functions $g_{1 i}\left(u_{11}, u_{12}, u_{13}, u_{14} ; \epsilon\right)$ are omitted for brevity. The corresponding Jacobian matrix of equations evaluated at the equilibrium $u_{2 i}=0(i=1,2,3,4)$ is in the Jordan canonical form $J_{H 2}=\operatorname{diag}\left[\left[\begin{array}{cc}0 & \omega_{20} \\ -\omega_{20} & 0\end{array}\right],-0.1605,-0.0103\right]$, where $\omega_{20}=0.0948$. The corresponding Hopf bifurcation normal form up to third order term is written as $\frac{d r_{2}}{d t_{2}}=r_{2}\left(v_{10} \epsilon+v_{21} r_{2}^{2}\right)$ and $\frac{d \theta_{2}}{d t_{2}}=\omega_{20}+\tau_{20} \epsilon+\tau_{21} r_{2}^{2}$, where $v_{20}=-0.0034, \tau_{20}=-0.0034, v_{21}=-0.1238 \times 10^{-8}$, and $\tau_{21}=-0.1584 \times 10^{-9}$. The trivial equilibrium $\bar{r}_{20}=0$ indicating $E_{H 2}$ is stable if $\epsilon>0$ and unstable if $\epsilon<0$, while the non-trivial equilibrium $\bar{r}_{21}=-\frac{v_{20} \epsilon}{v_{21}}$ exists and is stable if $\epsilon<0$. A stable limit cycle is generated from this Hopf bifurcation.

\section{Demonstrating roles of macrophage programmed cell death through numerical simulations}

The bifurcation diagrams in Fig. 3 demonstrate the diverse Mtb levels $B$ with regard to the infected macrophage programmed cell death (apoptosis) rate $b$. Disease-free equilibrium $E_{0}$ (red line in Fig. $3 \mathrm{~b}$ ) and infected equilibrium $E^{*}$ (blue curve in Fig. $3 \mathrm{a}, \mathrm{b}$ ) intersect and exchange stability at a transcritical bifurcation (marked as point $\mathrm{BP}$ at $b=b_{T 4}$ ). In the first branch from the bottom of the infected equilibrium, $E^{*}$ is unstable and undergoes two neutral saddle points (marked as NS), then bifurcates to a stable equilibrium solution in the second branch from the bottom at a saddlenode bifurcation (marked as $\mathrm{LP}_{1}$ at $b=b_{T 1}$ ). The stable $E^{*}$ loses its stability at a supercritical Hopf bifurcation at $b=b_{H 1}$ (marked as $\mathrm{H}_{1}$ ). The stable bifurcating limit cycles disappear when the periodic solution merges with the lowest branch of $E^{*}$ (see Fig. 3b) and induces a blow up in the period (see Fig. 3c top). $E^{*}$ keeps being unstable until reaching to a second supercritical Hopf bifurcation at $b=b_{H 2}$ (marked as $\mathrm{H}_{2}$ ). The corresponding bifurcating stable limit cycles also vanish when the cycles merge with the lowest branch of $E^{*}$ (see Fig. $3 b$ ), and induce a blow up in the period (see Fig. 3c bottom). This leaves a window in the branch at the second from the bottom of $E^{*}$ (approximately $b \in\left(0.052,0.163\right.$ day $^{-1}$ ), where solutions converge to the stable disease-free equilibrium $E_{0}$ (red line). Bistability is possible if $b \in\left(b_{T 3}, 0.163\right)$ day $^{-1}$. The stable branch at the second from the bottom of $E^{*}$ after $\mathrm{H}_{2}$ bifurcates an unstable branch (at the second from the top) at another saddle node bifurcation (marked as $\mathrm{LP}_{2}$ ) at $b=b_{H 2}$. Then $E^{*}$ gains back stability at the third 


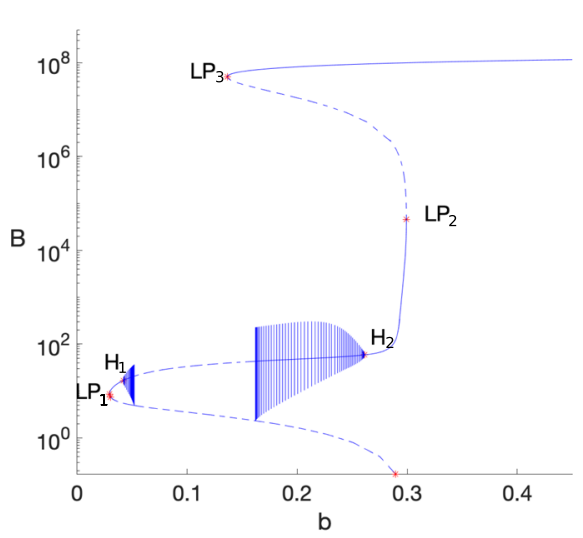

(a) Bifurcation diagram: $b$ vs $B$

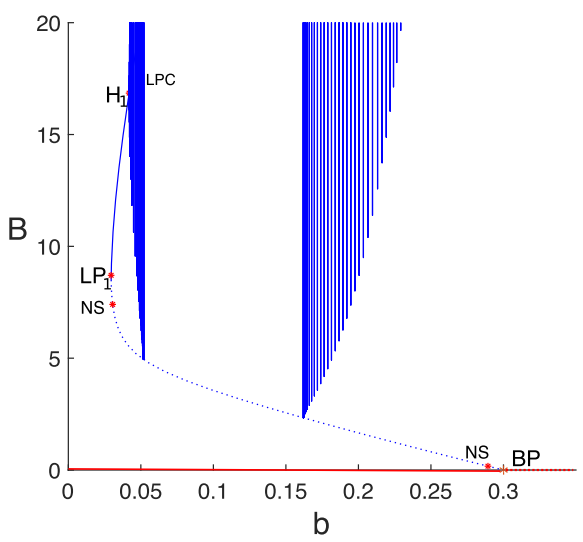

(b) A closeup of (a)
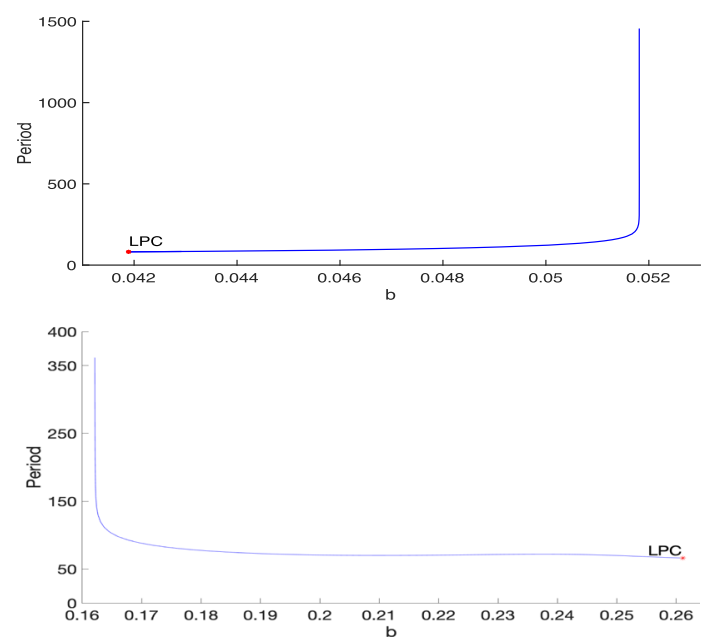

(c) Period vs $b$

Fig. 3 Bifurcation diagram of Mtb concentration $B\left(y_{3}\right)$ vs macrophage apoptosis rate $b$. Infected $\left(E^{*}\right)$ equilibriums are plotted according to Equation 1 in Appendix (see supplementary material) as $F\left(y_{3}, b\right)=0$ in blue lines (see (a) and (b)). The disease-free $\left(E_{0}\right)$ is in red. The solid and broken line styles distinguish stable and unstable equilibriums. Oscillating bacterial load are due to two supercritical Hopf bifurcations. Two oscillation regions are separated because periodic solutions merge with the lowest branch of $E^{*}$ (see (b)) and generate blow-up periods (see (c)) (color figure online)

saddle node bifurcation (marked as $\mathrm{LP}_{3}$ ) at $b=b_{H 3}$. It yields the top stable branch for the infected equilibrium $E^{*}$. Overall, the model (1) admits various disease outcomes (clearance, latent infection (LTBI), and active infection Cadena et al. 2017) through the occurrence of three saddle node bifurcations $\left(\mathrm{LP}_{1}\right.$ at $b=b_{T 1}, \mathrm{LP}_{2}$ at $b=b_{T 2}$, $\mathrm{LP}_{3}$ at $b=b_{T 3}$ and $\mathrm{BP}$ at $\left.b=b_{T 4}\right)$. Interestingly, in the second $E^{*}$ branch from the bottom, where Mtb level $B$ is in latent stage $\left(b \in\left(P L_{1}, L P_{2}\right)\right)$, the Mtb level first stabilizes at a low level $\left(b \in\left(L P_{1}, H_{1}\right)\right)$, then oscillates $b \in\left(H_{1}, 0.052\right)$, then vanished $\left(b \in(0.052,0.163)\right.$ day $\left.^{-1}\right)$, and oscillate again $\left(b \in\left(0.163, b_{H 2}\right)\right.$ day $\left.^{-1}\right)$, 
then gain back stability ( $b \in\left(H_{1}, L P_{2}\right)$ ). These surprisingly complex dynamical behaviors motivate us to investigate the macrophage-Mtb interaction model 1 regarding the macrophage apoptosis against Mtb.

\subsection{Macrophage programmed cell death in TB infection}

Before interpreting the complex macrophage-Mtb interplay shown in the bifurcation diagrams in Fig. 3 and the simulations in Figs. 4 and 5, it may be helpful to review macrophage apoptosis as a defense strategy against Mtb infection. Host immune responses to tuberculosis infection start from the inhalation of air droplets containing Mtb pathogens. These bacilli first manage to enter into resident alveolar macrophages through the phagocytosis process. A phagolysosome is then formed by the fusion of lysosomes with phagosomes containing engulfed Mtb pathogens. In the phagolysosome, internalized bacilli are degraded by reactive oxygen species and the acidification. However, Mtb pathogens evolve to survive from this cell anti-bacterial mechanism and to reproduce inside the macrophage. At this point, infected macrophages prohibit the extracellular Mtb pathogens, but facilitate intracellular bacterial replication at least until the activation of the adaptive immune responses (Sturgill-Koszycki et al. 1994), and provide an intracellular environment in favor of bacterial latency (Kornfeld et al. 1999). To defend against intracellular parasitic Mtb pathogens, a common strategy is to initiate host cell apoptosis. It destroys the protected intracellular environment, which promote intracellular bacterial reproduction and latency (Lee et al. 2009). However, to confront the elimination through host cell apoptosis, Mtb pathogens (especially virulent Mtb strains) evolve to interrupt tumor necrosis factor- $\alpha$ (TNF- $\alpha$ ) signaling (Spira et al. 2003) and promote anti-apoptotic Mcl-1 (Sly et al. 2003). These signals suppress the infected macrophage apoptosis. On the other hand, after maintaining the suitable intracellular environment, virulent strain Mtb pathogens manage to replicate, but still need a mean to spread the newly replicated pathogens. Host cell death is then triggered by heavy load of intracellular bacteria to spread the infection to other host cells. This heavily infected macrophage apoptosis process progresses rapidly and ends up in macrophage necrosis. It is found that the intracellular Mtb pathogens released from this process are not killed by host immune cells and able to replicate as extracellular bacteria (Lee et al. 2006). The macrophage necrosis caused by heavy load of intracellular Mtb benefits the TB progression to active TB disease.

\subsection{TB outcomes with a small bacterial load}

Pathogen clearance is possible if $b<b_{T 4}$, due to the local stability of the disease free equilibrium $E_{0}$. To represent the low bacterial level due to low inhaled bacterial load or antibiotic drug treatments, we choose a low initial bacterial load $\left(M_{u}(0), M_{i}(0), B(0), T(0)\right)=(10,1,1,100)$ cells/ml for the simulations in Fig. 4. The simulated Mtb infection clearance are shown in Fig. 4 panel (a) for six different macrophage apoptosis rates satisfying $b<b_{T 4}$ day $^{-1}$. Simulations in Fig. 4a also demonstrate a positive relation between infection clearance time (and the Mtb peak magnitude) and infected macrophage apoptosis rate $b$. It is probably because the 


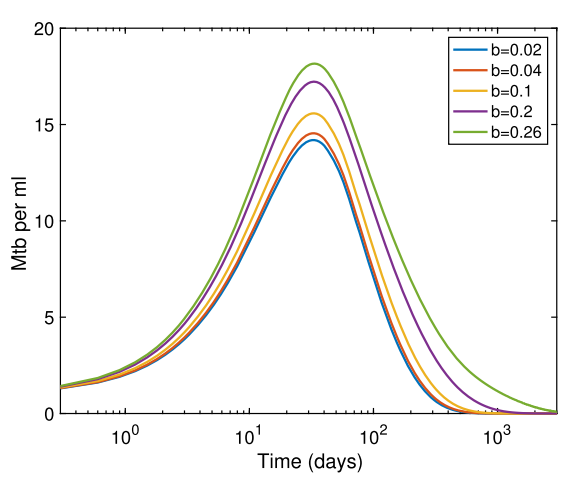

(a) Clearance after exposure

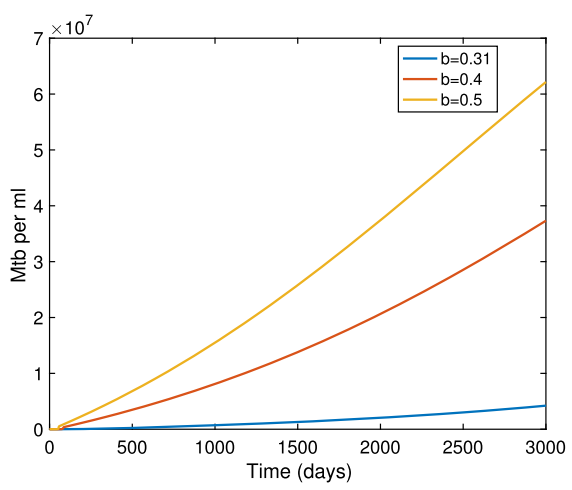

(b) Primary infection after exposure

Fig. 4 Clearance and development of infection with different speeds

release of intracellular Mtb pathogens during the Mtb-containing macrophage apoptosis process causes the increase of the total bacterial load, which takes the immune system longer time to eliminate.

Increasing the Mtb-containing macrophage apoptosis rate passing $b>b_{T 4}$ day $^{-1}$ indicates the occurrence of heavily infected macrophage necrosis. In this scenario, the host immune system loses control of the growth of the invading pathogen. Either a small amount of inhaled Mtb pathogens or a small load of latent Mtb bacteria will eventually progress to active TB disease (see Fig. $4 \mathrm{~b}$ ). A positive relation between the speed (and magnitude) and infected macrophages apoptosis rate $b$ is also observed in Fig. 4b. This result supports the experimental finding that the release of Mtb pathogens through necrosis induces the high Mtb bacterial burden (Lee et al. 2011).

Overall, simulation results confirm the experimental findings that the infected macrophage undergoing apoptosis promotes the clearance of Mtb infection (Keane et al. 1997, 2000; Lee et al. 2009) even though the released Mtb pathogens increase the total bacterial burden and result in longer clearance time. Moreover, the apoptosis for heavily infected macrophages culminates in necrosis, which releases more intracellular Mtb pathogens (Lee et al. 2009). The uncontrolled infection prolongs clearance time and facilitates infection progression to active TB disease.

\subsection{The tug of war between macrophage apoptosis and TB infection in LTBI state}

Latent tuberculosis infection (LTBI) describes an Mtb-infected individual who does not develop active disease and is not contagious. In this stage, the Mtb-infected macrophages provide a suitable environment for the latency and persistence of intracellular bacteria. However, diseases impairing the immune system (such as AIDS), treatments causing immune system suppression (such as chemotherapy for cancer), or advanced age can trigger LTBI reactivation. The LTBI status is demonstrated as low levels of Mtb pathogen load in Fig. 3 when the macrophage apoptosis rate is between $\mathrm{LP}_{1}$ and $\mathrm{LP}_{2}$. Low levels of Mtb load can be both persistent ( $b$ between $\mathrm{LP}_{1}$ and $H_{1}$ or between $H_{2}$ and $\mathrm{LP}_{2}$ ) and oscillatory (generated from two Hopf bifurcations $H_{1}$ 


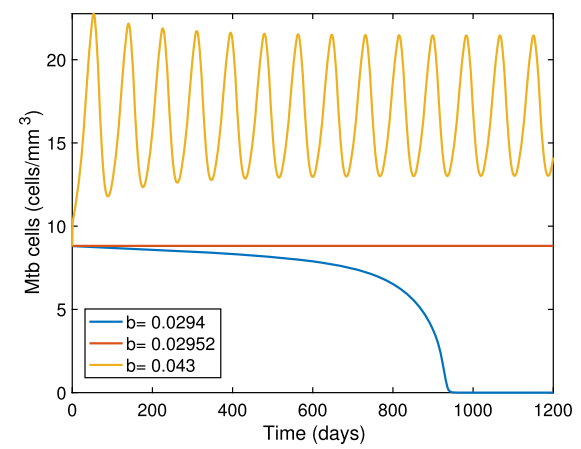

(a) Pathogen clearance and Mtb persistence

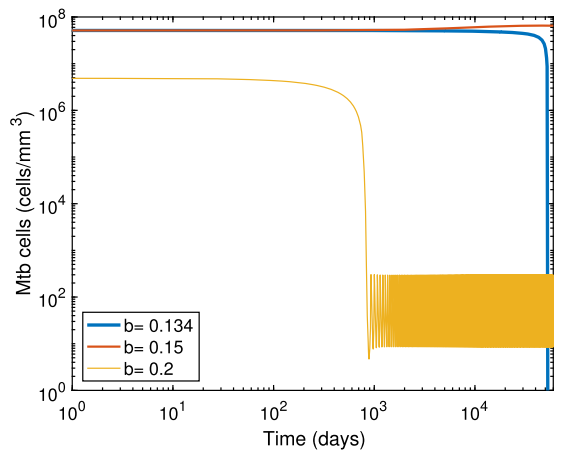

(c) Apoptosis progresses to necrosis

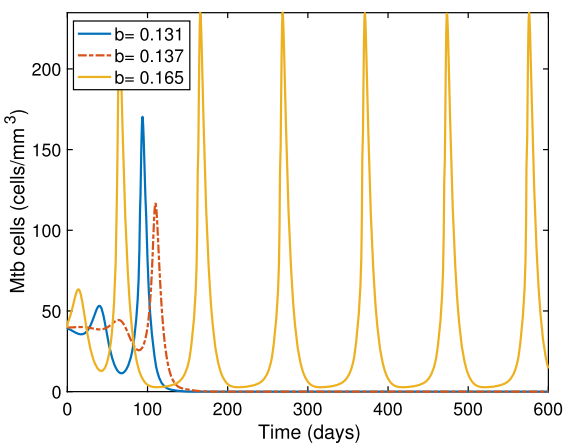

(b) Apoptosis benefits clearance

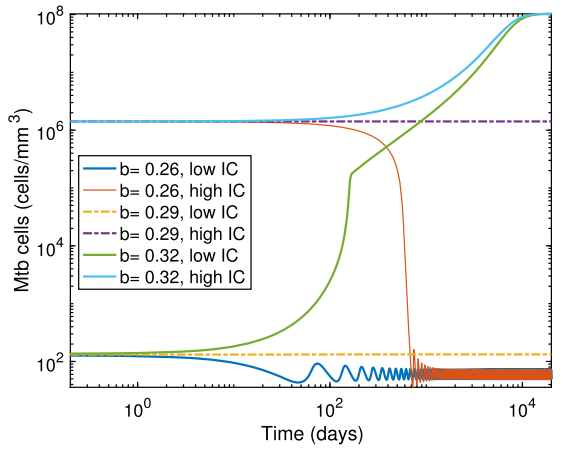

(d) Controllable and in controllable necrosis

Fig. 5 Simulated Mtb concentration as the result of the interplay between the host defense mechanism (macrophage apoptosis) and virulent Mtb strains counteract strategies (inhibition of macrophage apoptosis and trigger macrophage necrosis)

and $H_{2}$ ). Interestingly, the oscillations won't persist in the whole parameter interval of $\left(H_{1}, H_{2}\right)$, but disappear when oscillations starting from $H_{1}$ and starting from $H_{2}$ merge with the lower branch of the infected equilibrium $E^{*}$ (see Fig. 3b). It follows blowing-up oscillation periods (see Fig. 3c), where the coexisting unstable branches of infected equilibrium $E^{*}$ repel nearby solutions to the locally asymptotically stable disease free equilibrium $E_{0}$. A macrophage apoptosis rate window (approximately $\left(0.052,0.163\right.$ day $\left.^{-1}\right)$ between two Hopf bifurcations $H_{1}$ and $H_{2}$ are generated, where oscillations disappear. Moreover, the risk to progress to active disease is possible if the macrophage apoptosis rate $b$ is between $\mathrm{LP}_{3}$ and $\mathrm{LP}_{2}$, and if large load of Mtb pathogens is released due to the weakened immune system.

The role of macrophage apoptosis on the Mtb pathogen dynamics can be demonstrated by time-history simulations (see Fig. 5) based on the analytical predictions in bifurcation diagrams in Fig. 3. The clearance of internalized Mtb pathogens after phagocytosis by the acidification is demonstrated (see Fig. 5a) by a low level of macrophage apoptosis rate $b$ near $\operatorname{LP}_{1}\left(b<b_{T 1}\right.$ and $b=0.0294$ day $\left.^{-1}\right)$ and a low initial Mtb concentration in LTBI state. To confront the parasite pathogen after the infection establishment, Mtb-containing macrophages adopt a defense strategy to 
activate the host cell programmed death (macrophage apoptosis). This host defense mechanism is demonstrated by increasing the macrophage apoptosis rate $b$ to the window (approximately $\left(0.052,0.163\right.$ day $\left.^{-1}\right)$. Disease clearance is shown in both LTBI case (with low initial Mtb load) for $b=0.131 \mathrm{day}^{-1}$ and $b=0.1317 \mathrm{day}^{-1}$ ) (see Fig. 5b) and high initial Mtb load case for $b_{T 3}>b=0.134$ day $^{-1}$ (see Fig. 5c). Infected macrophage apoptosis effectively inhibits intracellular Mtb reproduction. To counteract this survival disadvantage, Mtb pathogens evolve virulent strains, which are capable of suppressing Mtb-containing macrophage apoptosis and continuing intracellular replication. The reduced macrophage apoptosis rate by virulent $\mathrm{Mtb}$ strains is between $b_{T 1}=0.0295 \mathrm{day}^{-1}\left(\mathrm{LP}_{1}\right)$ and $0.163 \mathrm{day}^{-1}$. The low level persistent and oscillatory Mtb levels are shown in Fig. 5a for $b_{T 1}<b=0.02952$ day $^{-1}$ and $0.043 \mathrm{day}^{-1}$ ). Virulent Mtb strains manage to suppress macrophage apoptosis for the intracellular replication, but also provoke host macrophage necrosis when the intracellular pathogen load passes a threshold or the host cell resources are depleted. The rapid macrophage apoptosis progresses to macrophage necrosis, which induces a large amount of intracellular pathogen release and benefits the infection. Figure $5 \mathrm{~b}$ and $\mathrm{c}$ show that the low-level oscillatory Mtb level for $b=0.165 \mathrm{day}^{-1}$ and $b=0.2$ day $^{-1}\left(\in\left(0.043, H_{2}\right)\right.$ day $\left.^{-1}\right)$ and an active disease case for $b=0.15$ day $^{-1}$ $\left(\in\left(b_{T 3}, 0.043\right)\right.$ day $\left.^{-1}\right)$ if the patient is exposed with high level Mtb pathogen. Even undergoing host cell necrosis, the immune system can still manage to control the growth of Mtb pathogens (shown in Fig. 5 for $b=0.26$ day $^{-1}$ and $b=0.29$ day $^{-1}$ ), but fails if the infected macrophage loss rate passes through the threshold $b_{T 4}(\mathrm{BP})$ (shown in Fig. 5 for $b=0.32$ day $^{-1}$ ).

Our analytical and numerical results confirm the mechanism that the host immune system employs programmed cell death (apoptosis) as a strategy to defend against intracellular Mtb parasitism (Lee et al. 2009). However, Mtb pathogens counteract macrophage apoptosis by evolving virulent Mtb strains. Virulent Mtb strains can both inhibit macrophage apoptosis to facilitate intracellular Mtb replication and trigger heavily-infected macrophage necrosis to release intracellular pathogens (Zychlinsky 1993; McCormick 2008; Lee et al. 2009). This complex parasitic relationship determines the various TB disease outcomes. Moreover, a comprehensive understanding of the interplay between macrophage apoptosis and Mtb pathogens is fundamental for the TB treatment design.

\section{New treatments suggested by the new insights of macrophages programmed cell death}

The current standard treatment of TB involves four first-line antibiotics (WHO 2019). Effective treatment for curing TB, however, can be hampered by the development of drug resistance, which can occur when treatment is not properly completed or adhered to. Host directed therapy (HDT), which includes small molecule therapies that can modulate the host immune response, can be used to augment treatment regimens to better control TB infection of both antibiotic sensitive and resistant TB strains (Kolloli and Subbian 2017). Antibiotic and HDT treatment can increase bacterial elimination $(\eta)$, infected macrophage loss $(b, \gamma)$, T-cell generation $\left(s_{T}, c_{m}, c_{b}\right)$, and decrease 


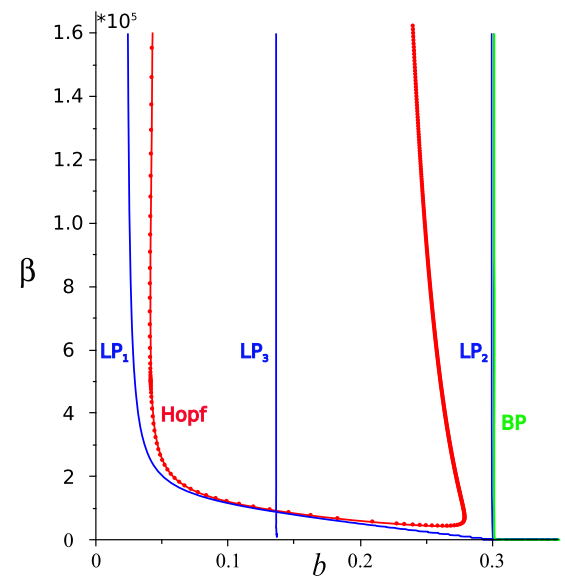

(a)

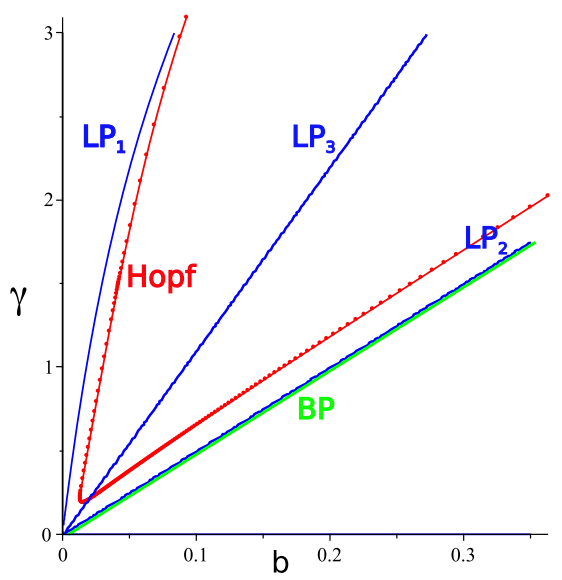

(c)

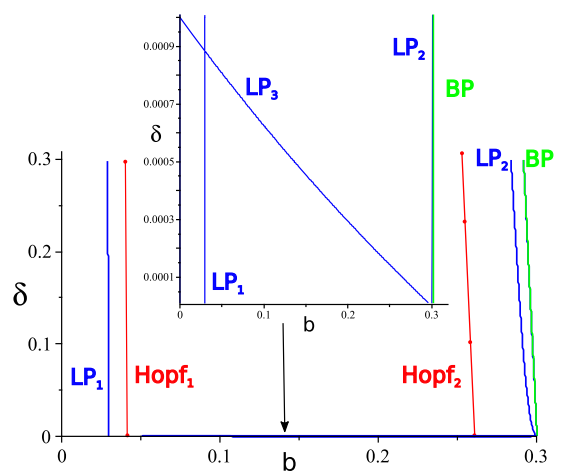

(b)

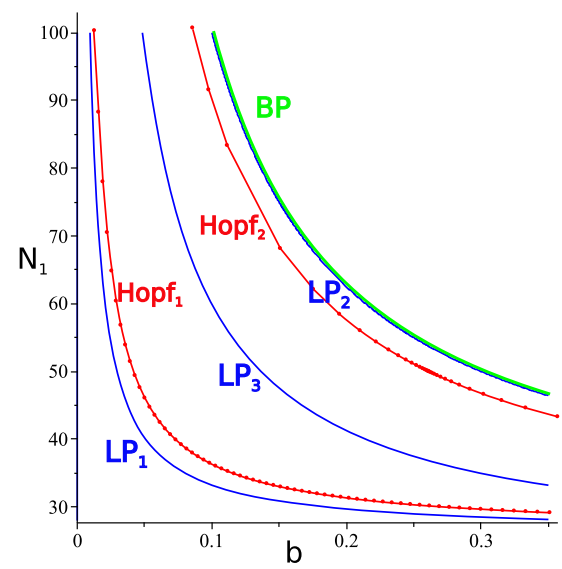

(d)

Fig. 6 2-dimensional bifurcation diagrams of $b$ vs $\beta, \delta, \gamma$ and $N_{1}$

macrophage infection $(\beta)$, and intracellular and extracellular bacterial proliferation $(\delta$, $N_{1}, N_{2}$ ). Sensitivity analysis has shown that $\eta, s_{T}, c_{m}, c_{b}$, and $N_{2}$ do not significantly affect the reproduction number (Fig. 1) or LTBI reactivation (Fig. 2). These parameters are therefore ignored, and we proceed with an analysis of $b, \beta, \delta$, and $\gamma$ and $N_{1}$, which significantly affect the reproduction number and LTBI reactivation.

Figure 6 plots two parameter bifurcation diagrams that consider parameter $b$ with $\beta, \delta, \gamma$, and $N_{1}$. For convenience in the bifurcation analyses, we denote the TB model (1) as $\frac{d x}{d t}=f(x, p)$, where $x=\left(M_{u}, M_{i}, B, T\right) \in R^{4}, p=\left(p_{b}, p_{f}\right) \in R^{18}$, and $f: R^{4+18} \rightarrow R^{4}$. Here, $p_{b} \in R^{2}$ denotes the two chosen bifurcation parameters, which are one of the following pairs: $(b, \beta),(b, \delta),(b, \gamma)$, and $\left(b, N_{1}\right) . p_{f}$ represents the remaining 16 parameters, which take the corresponding fixed values from Table 1 . We represent equilibrium solutions as $x_{e}=x_{e}\left(p_{b}\right)$. Single-zero eigen- 
value bifurcation happens if $\operatorname{det}\left(J\left(x_{e}\left(p_{b}\right), p_{b}\right)\right)=0$ with positive Hurwitz conditions $\Delta_{i}>0$, for $i=1,2,3$. Hopf bifurcation occurs when (1) $\left.\Delta_{3}\left(x_{e}\left(p_{b}\right), p_{b}\right)\right)=0$, (2) $\Delta_{1,2}\left(x_{e}\left(p_{b}\right), p_{b}\right)>0,(3) \operatorname{det}\left(J\left(x_{e}\left(p_{b}\right), p_{b}\right)\right)>0$ (positive determinant of the corresponding Jacobian matrix), and (4) $\frac{d \Delta_{3}\left(x_{e}\left(p_{b}\right), p_{b}\right)}{d p_{b}} \neq 0$. Single-zero (saddle-node) bifurcation curves are denoted as LP in blue. Transcritical bifurcation satisfying (4) are denoted as BP in green. Hopf bifurcation curves are plotted in red. Here, $\mathrm{LP}_{1}, \mathrm{LP}_{2}$, and $\mathrm{LP}_{3}$ correspond to the three limit point (saddle-node) bifurcations in Fig. 3a. BP is consistent with the branching point (transcritical) bifurcation and Hopf matches with the two Hopf bifurcations. Note that $\mathrm{LP}_{1}$ and $\mathrm{LP}_{3}$ denote two different branches of equilibrium solutions in Fig. 3a. Therefore, even though the parameter curves for $\mathrm{LP}_{1}$ and $\mathrm{LP}_{3}$ intersect (see Fig. 6) in the corresponding 2-dimensional parameter space, the corresponding equilibrium solutions are different. Therefore, there is no intersection in the parameter-state space. It follows that no higher-codimension bifurcation occurs. We note that Bogdanov-Takens, generalized Hopf, and zero-Hopf bifurcations are detected for the bifurcation analyses $b$ vs $\gamma$ and $b$ vs $\delta$. However, the bifurcation critical points lie outside of biologically feasible regions from Table 1, and therefore, are not considered.

Figure 6 first shows that with a fixed value for $\beta, \delta, \gamma$, and $N_{1}$, the order of bifurcation occurrence matches the order shown in Fig. $3 \mathrm{a}-$ as $b$ increases, we have $\mathrm{LP}_{1}, \mathrm{Hopf}_{1}$, $\mathrm{LP}_{3}, \mathrm{Hopf}_{2}, \mathrm{LP}_{3}$, and BP. Panels (a) and (b) also show that as $b$ changes, the infection outcome does not vary widely, given change in $\beta$ and $\delta$. Therefore, if drug therapy moderates the macrophage apoptosis rate $b$, very large changes in Mtb infection rate $\beta$ or Mtb proliferation rate $\delta$ are needed to see a change from a current stable equilibrium or periodic cycle. Additionally, to achieve bacteria elimination and prevent reactivation of LTBI, for a given $\beta$ or $\delta, b$ must be decreased to a value smaller than $\mathrm{LP}_{2}$. As the macrophage apoptosis rate $b$ changes, changes in cell-mediated immunity rate $\gamma$ and average number of released bacteria $N_{1}$ can also have an effect. Here, we identify the disease clearance region (in Fig. 6 above $\mathrm{LP}_{1}$ in (c) or below $\mathrm{LP}_{1}$ in (d)) and LTBI controllable region (in Fig. 6 above $\mathrm{LP}_{2}$ in (c) or below $\mathrm{LP}_{2}$ in (d)). To enlarge the disease clearance and LTBI controllable regions, we need to increase $b$ and increase $\gamma$ or decrease $N_{1}$. It means that a drug therapy that moderates the macrophage apoptosis works better with the combination of an increased killing rate of infected cells by the immune system $\gamma$ or a decreased Mtb released number $N_{1}$ during programmed cell death.

In drug development, research on apoptosis modulation indicates therapeutic value in cancer (Reed 2004), cardiovascular disease, and other diseases (Cotter et al. 2003). Our findings enhance the idea that a drug therapy promoting macrophage apoptosis, but inhibiting the progression to necrosis induced by heavily-infected cell death, could benefit host defense against Mtb. In the mean time, we should consider the pathogen anti-apoptosis strategies, which could increase bacillary viability, then increase the number of released intracellular Mtb $N_{1}$. For example, Mtb virulent strains are capable of inhibiting host macrophage apoptosis for intracellular reproduction by hindering the tumor necrosis factor $\alpha(\mathrm{TNF} \alpha)$. A negative therapeutic effect is associated with treating TB with infliximab, a TNF $\alpha$-neutralizing agent (Keane et al. 2001). Moreover, adaptive immunity is promoted by the effect of the efferocytosis process, in which 
Mtb-infected apoptotic corpses are removed by phagocytic cells. These phagocytic cells then present Mtb antigen and active CD8+ T cells. This phenomenon not only explains the positive effect of macrophage apoptosis on host, but also inspires vaccine development to promote adaptive immunity (Lee et al. 2009).

In conclusion, to prevent LTBI reactivation and to eliminate TB disease, the development of apoptosis modulation drugs must take the Mtb survival strategies into account. Furthermore, an understanding of the enhanced cell-mediated immunity gives new insight into vaccine development.

\section{Conclusion}

In this paper, we investigate the macrophage and Mycobacterium tuberculosis (Mtb) dynamics through an established 4-dimensional host-pathogen model. We pay particular attention to identify the determining mechanisms for Mtb elimination, LTBI reactivation prevention, and active TB treatment.

The first goal is to identify the determining factors for various disease outcomes that are unique features for TB infection. These include clearance, LTBI, active disease, and LTBI reactivation. Various disease outcomes in the mathematical model indicate the co-existing Mtb steady states levels (equilibriums). The basic mathematical mechanism for the creation and emergence of steady states is the occurrence of saddle-node bifurcation. Additionally, the fundamental mathematical mechanism for the disease clearance and persistence is the occurrence of transcritical bifurcation. Both saddle-node and transcritical bifurcations are single zero-eigenvalue bifurcation. The problem of identifying parameter sensitivity for TB elimination and LTBI reactivation is replaced by the problem of identifying parameter sensitivity for the occurrence of single zero-eigenvalue bifurcation. Note that pitchfork bifurcation is the other single zero-eigenvalue bifurcation, but is not common in infectious disease models due to its symmetric nature. It is therefore left out of our consideration.

We then perform a sample-based sensitivity analysis for the condition of transcritical bifurcation (the basic reproduction number) on all eleven model parameters. Our results demonstrate that eight of the eleven parameters significantly influence the basic reproduction number $\left(R_{0}\right)$. The eight parameters are the infection rate $\beta, \mathrm{T}$ cell-mediated immunity rate $\gamma$, extracellular bacterium proliferation rate $\delta$, infected macrophages apoptotic rate $b$, uninfected macrophages bacterial killing rate $\eta$, and average number of bacterium engulfed by uninfected macrophages $N_{3}$ or released through the death of infected macrophage induced by apoptosis $N_{1}$ and cell-mediated immunity $N_{2}$. Fixing the parameters that do not statistically significantly affect the basic reproduction number, another sensitivity analysis is carried out for the conditions of saddle node bifurcation on the eight parameters that significantly influence $R_{0}$. These two steps of sensitivity analyses find that the infected macrophage cell death rate $b$ is the most influential factor on both $R_{0}$ and the occurrence of saddlenode bifurcation, which determines the occurrence of various disease outcomes and, in turn, rules the fate of the disease. Biologically, the host cell death in the form of apoptosis is considered to inhibits Mtb replication and to produce bacterial antigens to activate adaptive immune responses (Behar et al. 2011; Queval et al. 2017). On the 
other hand, cell death in the form of necrosis is associated with spreading infection (Behar et al. 2011; Queval et al. 2017). Other proposed host factors and Mtb invading strategies, which contribute to the disease progression, include genetic diversity (Gagneux 2013), T-cell mediated immune responses (Verrall et al. 2014; Flynn and Chan 2001), the responses of two pivotal cytokines gamma interferon (IFN- $\gamma$ ) and tumor necrosis factor alpha (TNF- $\alpha$ ) (Flynn and Chan 2001), Mtb inhibiting the elimination of phagocytized bacteria Queval et al. (2017), and Mtb controlling host cell death to escape from kaltilling Queval et al. 2017. One limitation of our mathematical results is the choice of parameter values. The parameter ranges in Table 1 are based on current experimental and clinical literature, but still inherit their restrictions. Nevertheless, our mathematical results confirm the crucial and controversial role in the host immune defense against invading Mtb pathogens (Queval et al. 2017).

Sensitivity analysis dramatically narrows down parameters of interest for understanding model behaviors (including infection elimination and LTBI progression), but it can not tell how the identified parameters affect the model behaviors, or what model dynamics can be unfolded from the change of the identified parameters. Answering these two questions serves as our second goal. We employ bifurcation analysis to tackle these questions. Considering the macrophage apoptosis rate $b$ as a bifurcation parameter, we explore the role of macrophage apoptosis in TB infection through bifurcation analyses. A spectrum of disease outcomes are verified by numerical simulations. We carry out bifurcation analyses on single-zero eigenvalues bifurcations and derive Hopf bifurcation normal forms. Our analytical results are consistent with the numerical bifurcation output from MatCont (Dhooge et al. 2003).

Regarding TB pathobiology, our findings of the mathematical model dynamics agree with the clinical and experimental macrophage-Mtb interaction outcomes (Lee et al. 2009), which consider the complex roles of macrophage apoptosis in the host defense against TB infection. Our mathematical model (1) demonstrates the following: (1) the clearance of low level Mtb load during phagocytosis is associated with low macrophage apoptosis rate (Verrall et al. 2014); (2) the TNF $\alpha$-induced macrophage apoptosis facilitates the host defense against intracellular Mtb proliferation (Lee et al. 2009); (3) macrophage apoptosis is suppressed by virulent Mtb strains interfering with $\mathrm{TNF} \alpha$ signaling pathways. Virulent Mtb then gain an intracellular reproduction environment (Kornfeld et al. 1999); and (4) heavily infected macrophage apoptosis induces macrophage necrosis, which releases a large amount of intracellular bacteria and benefits the spread of the pathogen (Lee et al. 2009; Behar et al. 2011).

Elucidating the complex roles of macrophage apoptosis played in Mtb in-host dynamics could give new insights into the treatments aiming at TB elimination and LTBI reactivation prevention. Treatment strategies regulate macrophage apoptosis in combination with the control of bacillary viability is recommended. Mtb antigen presentation after the efferocytosis of Mtb-containing apoptotic remaining enhance adaptive immunity by activating CD8+ $\mathrm{T}$ cells. The adaptive immunity promoting mechanism can help the development of the new vaccine and new treatments.

Supplementary Information The online version contains supplementary material available at https://doi. org/10.1007/s00285-021-01655-6. 
Acknowledgements We acknowledge the financial support from Simons Foundation (A21-0013-001) and Texas Tech University (New Faculty Startup) for Wenjing Zhang, Canadian Network for Research and Innovation in Machining Technology, Natural Sciences and Engineering Research Council of Canada (Discovery Grant) and York University Research Chair for Jane Heffernan, and Australian Research Council (DP180101512) for Federico Frascoli. Conceptualization, methodology, formal analysis were carried out by Wenjing Zhang. The original draft of the manuscript was written by Wenjing Zhang and co-edited by Wenjing Zhang and Leif Ellingson. The investigation of the sensitivity analysis was performed by Wenjing Zhang and Leif Ellingson. The biological interpretation was carried out by Wenjing Zhang and Jane Heffernan. The revision is carried out by Wenjing Zhang and Leif Ellingson. All authors read and approved the final manuscript. The authors thank the editor and anonymous reviewers for their careful reading of our manuscript and their insightful comments and suggestions.

\section{References}

Abdelrazec A, Bélair J, Shan C, Zhu H (2016) Modeling the spread and control of dengue with limited public health resources. Math Biosci 271:136-145

Antia R, Koella JC, Perrot V (1996) Models of the within-host dynamics of persistent mycobacterial infections. Proc R Soc Lond B 263(1368):257-263

Barry CE, Boshoff HI, Dartois V, Dick T, Ehrt S, Flynn J, Schnappinger D, Wilkinson RJ, Young D (2009) The spectrum of latent tuberculosis: rethinking the biology and intervention strategies. Nat Rev Microbiol 7(12):845-855

Bauer AL, Hogue IB, Marino S, Kirschner DE (2008) The effects of HIV-1 infection on latent tuberculosis. Math Modell Nat Phenom 3(7):229-266

Behar SM, Martin CJ, Booty MG, Nishimura T, Zhao X, Gan HX, Divangahi M, Remold HG (2011) Apoptosis is an innate defense function of macrophages against mycobacterium tuberculosis. Mucosal Immunol 4(3):279-287

BoseDasgupta S, Pieters J (2014) Striking the right balance determines TB or not TB. Front Immunol 5:455

Cadena AM, Fortune SM, Flynn JL (2017) Heterogeneity in tuberculosis. Nat Rev Immunol 17(11):691-702

Canetti G (1955) The tubercle bacillus in the pulmonary Lesion of Man: Histobacteriology and Its Bearing on the Therapy of Pulmonary Tuberculosis. Springer Publishing Company, New York

Conover WJ, Iman RL (1981) Rank transformations as a bridge between parametric and nonparametric statistics. Am Stat 35(3):124-129

Cooper AM (2009) Cell-mediated immune responses in tuberculosis. Annu Rev Immunol 27:393-422

Cotter T, Murphy FJ, Seery LT, Hayes I (2003) Therapeutic approaches to the modulation of apoptosis. Essays Biochem 39:131-153

Dhooge A, Govaerts W, Kuznetsov YA (2003) Matcont: a MATLAB package for numerical bifurcation analysis of ODEs. ACM Trans Math Softw (TOMS) 29(2):141-164

Donoghue HD, Spigelman M, Greenblatt CL, Lev-Maor G, Bar-Gal GK, Matheson C, Vernon K, Nerlich AG, Zink AR (2004) Tuberculosis: from prehistory to Robert Koch, as revealed by ancient DNA. Lancet Infect Dis 4(9):584-592

Du Y, Wu J, Heffernan JM (2017) A simple in-host model for Mycobacterium tuberculosis that captures all infection outcomes. Math Popul Stud 24(1):37-63

Ehlers S, Schaible UE (2013) The granuloma in tuberculosis: dynamics of a host-pathogen collusion. Front Immunol 3:411

Flynn JL, Chan J (2001) Tuberculosis: latency and reactivation. Infect Immun 69(7):4195-4201

Gagneux S (2013) Genetic diversity in mycobacterium tuberculosis. Pathog Mycobacterium Tuberc Interact Host Organism, pages 1-25 (2013)

Gammack D, Ganguli S, Marino S, Segovia-Juarez J, Kirschner DE (2005) Understanding the immune response in tuberculosis using different mathematical models and biological scales. Multiscale Model Simul 3(2):312-345

Gideon HP, Flynn JL (2011) Latent tuberculosis: what the host "sees"? Immunol Res 50(2-3):202-212

Gong C, Linderman JJ, Kirschner D (2015) A population model capturing dynamics of tuberculosis granulomas predicts host infection outcomes. Math Biosci Eng MBE 12(3):625

Guckenheimer J, Holmes P (2013) Nonlinear oscillations, dynamical systems, and bifurcations of vector fields, vol 42. Springer Science \& Business Media, Berlin 
Helton JC, Davis FJ (2003) Latin hypercube sampling and the propagation of uncertainty in analyses of complex systems. Reliab Eng Syst Saf 81(1):23-69

Iman RL, Helton JC (1988) An investigation of uncertainty and sensitivity analysis techniques for computer models. Risk Anal 8(1):71-90

Keane J, Balcewicz-Sablinska MK, Remold HG, Chupp GL, Meek BB, Fenton MJ, Kornfeld H (1997) Infection by Mycobacterium tuberculosis promotes human alveolar macrophage apoptosis. Infect Immun 65(1):298-304

Keane J, Remold HG, Kornfeld H (2000) Virulent Mycobacterium tuberculosis strains evade apoptosis of infected alveolar macrophages. J Immunol 164(4):2016-2020

Keane J, Gershon S, Wise RP, Mirabile-Levens E, Kasznica J, Schwieterman WD, Siegel JN, Braun MM (2001) Tuberculosis associated with infliximab, a tumor necrosis factor alpha-neutralizing agent. N Engl J Med 345(15):1098-1104

Kinchen JM, Ravichandran KS (2008) Phagosome maturation: going through the acid test. Nat Rev Mol Cell Biol 9(10):781-795

Kirschner D (1999) Dynamics of co-infection with M. tuberculosisand HIV-1. Theor Popul Biol 55(1):94109

Kleijnen JP, Helton JC (1999) Statistical analyses of scatterplots to identify important factors in large-scale simulations, 1: Review and comparison of techniques. Reliab Eng Syst Saf 65(2):147-185

Kolloli A, Subbian S (2017) Host-directed therapeutic strategies for tuberculosis. Front Med 4:171

Kornfeld H, Mancino G, Colizzi V (1999) The role of macrophage cell death in tuberculosis. Cell Death Differ 6(1):71-78

Lauffenburger D, Linderman J (1993) Cell surface receptor/ligand binding fundamentals. Receptors: models for binding, trafficking and signaling Oxford Press, New York, NY pp 9-72

Lee J, Remold HG, Ieong MH, Kornfeld H (2006) Macrophage apoptosis in response to high intracellular burden of Mycobacterium tuberculosis is mediated by a novel caspase-independent pathway. J Immunol 176(7):4267-4274

Lee J, Hartman M, Kornfeld H (2009) Macrophage apoptosis in tuberculosis. Yonsei Med J 50(1):1-11

Lee J, Repasy T, Papavinasasundaram K, Sassetti C, Kornfeld H (2011) Mycobacterium tuberculosis induces an atypical cell death mode to escape from infected macrophages. PLoS ONE 6(3):e18367

Lewinsohn D, Bement T, Xu J, Lynch D, Grabstein K, Reed S, Alderson M (1998) Human purified protein derivative-specific CD4+ t cells use both CD95-dependent and CD95-independent cytolytic mechanisms. J Immunol 160(5):2374-2379

Lin PL, Flynn JL (2010) Understanding latent tuberculosis: a moving target. J Immunol 185(1):15-22

Magombedze G, Garira W, Mwenje E (2006) Modelling the human immune response mechanisms to Mycobacterium tuberculosis infection in the lungs. Math Biosci Eng 3(4):661

Marino S, Kirschner DE (2004) The human immune response to Mycobacterium tuberculosis in lung and lymph node. J Theor Biol 227(4):463-486

Marino S, Kirschner DE (2016) A multi-compartment hybrid computational model predicts key roles for dendritic cells in tuberculosis infection. Computation 4(4):39

Marino S, Hogue IB, Ray CJ, Kirschner DE (2008) A methodology for performing global uncertainty and sensitivity analysis in systems biology. J Theor Biol 254(1):178-196

McCormick AL (2008) Control of apoptosis by human cytomegalovirus. Curr Top Microbiol Immunol 325:281-295. https://doi.org/10.1007/978-3-540-77349-8_16

McKay MD, Beckman RJ, Conover WJ (1979) Comparison of three methods for selecting values of input variables in the analysis of output from a computer code. Technometrics 21(2):239-245

O'Garra A, Redford PS, McNab FW, Bloom CI, Wilkinson RJ, Berry MP (2013) The immune response in tuberculosis. Annu Rev Immunol 31:475-527

Organization WH, et al (2017) Global tuberculosis report 2017

Orme IM, Robinson RT, Cooper AM (2015) The balance between protective and pathogenic immune responses in the TB-infected lung. Nat Immunol 16(1):57-63

Queval CJ, Brosch R, Roxane S (2017) The macrophage: a disputed fortress in the battle against mycobacterium tuberculosis. Front Microbiol 8:2284

Ray JCJ, Flynn JL, Kirschner DE (2009) Synergy between individual tnf-dependent functions determines granuloma performance for controlling Mycobacterium tuberculosis infection. J Immunol 182(6):3706-3717

Reed JC (2004) Apoptosis mechanisms: implications for cancer drug discovery. Oncology (Williston Park, NY) 18(13 Suppl 10):11-20 
Rich AR et al. (1951) The pathogenesis of tuberculosis. The Pathogenesis of Tuberculosis (Edn 2)(1951)

Russell DG, Barry CE, Flynn JL (2010) Tuberculosis: what we don't know can, and does, hurt us. Science 328(5980):852-856

Saltelli A, Annoni P (2011) Sensitivity analysis. Springer, Berlin Heidelberg, pp 1298-1301

Saltelli A, Sobol IM (1995) About the use of rank transformation in sensitivity analysis of model output. Reliab Eng Syst Saf 50(3):225-239

Segovia-Juarez JL, Ganguli S, Kirschner D (2004) Identifying control mechanisms of granuloma formation during M. tuberculosis infection using an agent-based model. J Theor Biol 231(3):357-376

Sly LM, Hingley-Wilson SM, Reiner NE, McMaster WR (2003) Survival of mycobacterium tuberculosis in host macrophages involves resistance to apoptosis dependent upon induction of antiapoptotic BCL-2 family member MCL-1. J Immunol 170(1):430-437

Spira A, Carroll JD, Liu G, Aziz Z, Shah V, Kornfeld H, Keane J (2003) Apoptosis genes in human alveolar macrophages infected with virulent or attenuated Mycobacterium tuberculosis: a pivotal role for tumor necrosis factor. Am J Respir Cell Mol Biol 29(5):545-551

Sturgill-Koszycki S, Schlesinger PH, Chakraborty P, Haddix PL, Collins HL, Fok AK, Allen RD, Gluck SL, Heuser J, Russell DG (1994) Lack of acidification in Mycobacterium phagosomes produced by exclusion of the vesicular proton-atpase. Science 263(5147):678-681

Sud D, Bigbee C, Flynn JL, Kirschner DE (2006) Contribution of cd8+ t cells to control of Mycobacterium tuberculosis infection. J Immunol 176(7):4296-4314

Torrado E, Cooper AM (2013) Cytokines in the balance of protection and pathology during mycobacterial infections. Adv Exp Med Biol 783:121-140. https://doi.org/10.1007/978-1-4614-6111-1_7

Ulrichs T, Kaufmann SH (2006) New insights into the function of granulomas in human tuberculosis. J Pathol J Pathol Soc Great Br Ireland 208(2):261-269

Upadhyay S, Mittal E, Philips JA (2018) Tuberculosis and the art of macrophage manipulation. Pathog Disease 76(4):fty037

Verrall AJ, Netea MG, Alisjahbana B, Hill PC, van Crevel R (2014) Early clearance of m ycobacterium tuberculosis: a new frontier in prevention. Immunology 141(4):506-513

Warner DF, Mizrahi V (2007) The survival kit of Mycobacterium tuberculosis. Nat Med 13(3):282-284

WHO: Golbal tuberculosis report 2019. World Health Organization (2019)

Wigginton JE, Kirschner D (2001) A model to predict cell-mediated immune regulatory mechanisms during human infection with Mycobacterium tuberculosis. J Immunol 166(3):1951-1967

Wm Liu (1994) Criterion of Hopf bifurcations without using eigenvalues. J Math Anal Appl 182(1):250-256

Yu P, Wang X (2019) Analysis on recurrence behavior in oscillating networks of biologically relevant organic reactions. Math Biosci Eng 16(5):5263-5286

Yu P (2005) Closed-form conditions of bifurcation points for general differential equations. Int J Bifurc Chaos 15(04):1467-1483

Yu P, Wang X (2019) Analysis on recurrence behavior in oscillating networks of biologically relevant organic reactions. Math Biosci Eng 16:5263-5286

Zhang W, Frascoli F, Heffernan J (2020) Analysis of solutions and disease progressions for a within-host tuberculosis model. Math Appl Sci Eng 1:39-49

Zychlinsky A (1993) Programmed cell death in infectious diseases. Trends Microbiol 1(3):114-117

Publisher's Note Springer Nature remains neutral with regard to jurisdictional claims in published maps and institutional affiliations. 